\title{
Experimental Study of Tumor Therapy Mediated by Multimodal Imaging Based on a Biological Targeting Synergistic Agent
}

This article was published in the following Dove Press journal: International Journal of Nanomedicine

Yaotai Wang'
Chun Chen ${ }^{\prime}$
Yong Luo'
Jie Xiong'
Yu Tang'
Haiyan Yang'
Lu Wang'
Fujie Jiang'
Xuan Gao
Die Xu'
Huanan Li'
Qi Wang'
Jianzhong Zou

'State Key Laboratory of Ultrasound in Medicine and Engineering, College of Biomedical Engineering, Chongqing Medical University, Chongqing 400016, People's Republic of China; ${ }^{2}$ Chongqing Key Laboratory of Biomedical Engineering, Chongqing Medical University, Chongqing, 400016, People's Republic of China
Correspondence: Jianzhong Zou State Key Laboratory of Ultrasound in Medicine and Engineering, College of Biomedical Engineering, Chongqing Medical University, Yuzhong District, Chongqing 400016, People's Republic of China

Tel +86-13708302390

Email zoujzh@cqmu.edu.cn
Purpose: The high-intensity focused ultrasound (HIFU) ablation of tumors is inseparable from synergistic agents and image monitoring, but the existing synergistic agents have the defects of poor targeting and a single imaging mode, which limits the therapeutic effects of HIFU. The construction of a multifunctional biological targeting synergistic agent with high biosafety, multimodal imaging and targeting therapeutic performance has great significance for combating cancer.

Methods: Multifunctional biological targeting synergistic agent consisting of Bifidobacterium longum (B. longum), ICG and PFH coloaded cationic lipid nanoparticles (CL-ICG-PFH-NPs) were constructed for targeting multimode imaging, synergistic effects with HIFU and imagingguided ablation of tumors, which was evaluated both in vitro and in vivo.

Results: Both in vitro and in vivo systematical studies validated that the biological targeting synergistic agent can simultaneously achieve tumor-biotargeted multimodal imaging, HIFU synergism and multimodal image monitoring in HIFU therapy. Importantly, the electrostatic adsorption method and the targeting of B. longum to tumor tissues allow the CL-ICG-PFHNPs to be retained in the tumor tissue, achieve the targeting ability of synergistic agent. Multimodal imaging chose the best treatment time according to the distribution of nanoparticles in the body to guide the efficient and effective treatment of HIFU. CL-ICG-PFH-NPs could serve as a phase change agent and form microbubbles that can facilitate HIFU ablation by mechanical effects, acoustic streaming and shear stress. This lays a foundation for the imaging and treatment of tumors.

Conclusion: In this work, a biological targeting synergistic agent was successfully constructed with good stability and physicochemical properties. This biological targeting synergistic agent can not only provide information for early diagnosis of tumors but also realize multimodal imaging monitoring during HIFU ablation simultaneously with HIFU treatment, which improves the shortcomings of HIFU treatment and has broad application prospects.

Keywords: high-intensity focused ultrasound, nanoparticles, photoacoustic imaging, fluorescence imaging, ultrasound imaging, image-guided therapy

\section{Introduction}

As a new noninvasive tumor treatment technology, high-intensity focused ultrasound (HIFU) has been widely used in the treatment of solid tumors and has broad application prospects. ${ }^{1-7}$ The therapeutic principle of HIFU is similar to that of a magnifying glass focusing light. ${ }^{8}$ Briefly, the HIFU technique utilizes high-energy focusing devices, which focus multiple low-energy ultrasound beams on the target tumor in a certain way to induce thermal, cavitation and mechanical effects, which 
can cause irreversible coagulative necrosis, finally achieving therapeutic effects. ${ }^{9-12}$ However, as the depth of treatment increases, the ultrasonic energy attenuates, and increasing the therapeutic power can lead to serious side effects, such as skin burns, nerve damage and transient pain. ${ }^{13-15}$ Various synergistic agents, such as lipiodol, hydroxyapatite, ultrasound microbubbles, liquid fluorocarbon nanoparticles, and mesoporous silica and sonosensitizers, have been used to try to increase the efficiency of HIFU ablation and minimize damage to normal tissue. ${ }^{16-19}$ Among them, phase-transitional lipid nanoparticles consisting of liquid fluorocarbon perfluorohexane (PFH) with a low boiling point $\left(56^{\circ} \mathrm{C}\right)$ and liquid-gas phase transition characteristics have been widely applied for HIFU ablation to increase the cavitation effect to kill tumor cells. ${ }^{20,21}$ Additionally, these lipid nanoparticles, which undergo the liquid-gas phase transition with acoustic or optical droplet vaporization methods, could be used in ultrasound (US)enhanced imaging. ${ }^{22,23}$ Although the use of lipid nanoparticles improves the current status of HIFU treatment, there are still some problems that limit the development of HIFU.

HIFU treatment is inseparable from image monitoring, but single ultrasound imaging makes it hard to meet the growing demand for HIFU treatment. ${ }^{24-27}$ Compared with single-mode imaging, multimodal imaging is more conducive for integrating the advantages of individual imaging and providing more accurate biological information for early diagnosis and HIFU therapeutic monitoring of the tumor. $^{28,29}$ Indocyanine green (ICG), as an FDA-approved agent, has been recognized as a near-infrared (NIR) dye for in vivo fluorescence imaging and has been widely developed as a photoacoustic contrast agent, photosensitizer and photothermal agent for PA and therapy. ${ }^{30,31}$

To achieve better multimodal imaging and therapeutic effects, another problem that urgently needs to be solved is the targeting of the synergistic agent. Therefore, the design of a highly targeted and efficient delivery system is the key to achieving precision imaging and treatment of solid tumors. In this study, Bifidobacterium longum (B. longum) was chosen as a vehicle for transporting the synergistic agent into the solid tumor for the following reasons: First, there are hypoxic areas in the center of solid tumors, and the facultative anaerobic properties of B. longum make it easy to concentrate and multiply in the anaerobic zone after entering the blood, achieving tumor targeting. Second, B. longum is a common probiotic in the intestine that does not have pathogenic or immunological side effects. ${ }^{32-34}$ Third, numerous studies have shown that $B$. longum can be used as a carrier to carry genes and contrast agents to the targeted tumor tissues for tumor imaging and therapeutic purposes. ${ }^{35-39}$

To solve the problems mentioned above, we proposed the concept of a biologically targeted synergistic agent (Figure 1). ICG and PFH coloaded cationic lipids (CLICG-PFH-NPs) were designed via a simple two-step emulsion method and linked to Bifidobacteria by electrostatic adsorption. The biologically targeted synergistic agent construction was verified in vitro, and its physicochemical properties were further shown. In the in vivo experiments (Figure 1B-D), predestination targeting technology was adopted. First, B. longum was injected into the body through the tail vein, and the characteristics of B. longumtargeted proliferation in the tumors were utilized to make $B$. longum the target of the nanoparticles in the tumors. Seven days later, sufficient $B$. longum were proliferated in the tumor tissue. Then, the CL-ICG-PFH-NPs were injected into the body, and the cationic lipid nanoparticles were guided to enrich in the tumor target area through electrostatic adsorption by virtue of the negative surface charge of $B$. longum to achieve targeted multimodal imaging of tumors, HIFU synergism and multimodal image monitoring in treatment with HIFU.

A biologically targeted synergistic agent has several advantages: 1) it has good biocompatibility; 2) it is not limited to only one type of tumor but also has a good ability to target multimodal imaging and synergistic HIFU treatment for most solid tumors; 3) it not only provides comprehensive imaging diagnosis information of the tumor but also realizes real-time monitoring during the treatment process, enable researchers to adjust the treatment plan according to the treatment situation in realtime; 4) the efficiency and safety of HIFU ablation tumor can be greatly improved.

\section{Materials and Methods Materials}

Lipids including 1,2-dipalmitoyl-sn-glycero-3-phosphocholine (DPPC), 1, 2-distearoyl-sn-glycero -3-phosphoethanolamine-N-[amino(polyethyleneglycol)-2000] (DSPE-PEG 2000-Amine) and DC-cholesterol hydrochloride (DCCHOL) were purchased from Avanti Polar Lipids (Alabaster, AL, USA). Chloroform $\left(\mathrm{CHCL}_{3}\right)$ was purchased from Chongqing East Chemical Industry Ltd, Co. (Chongqing, China). Indocyanine Green (ICG) was obtained from Aladdin (Shanghai, China). 2-(4-Amidinophenyl)- 


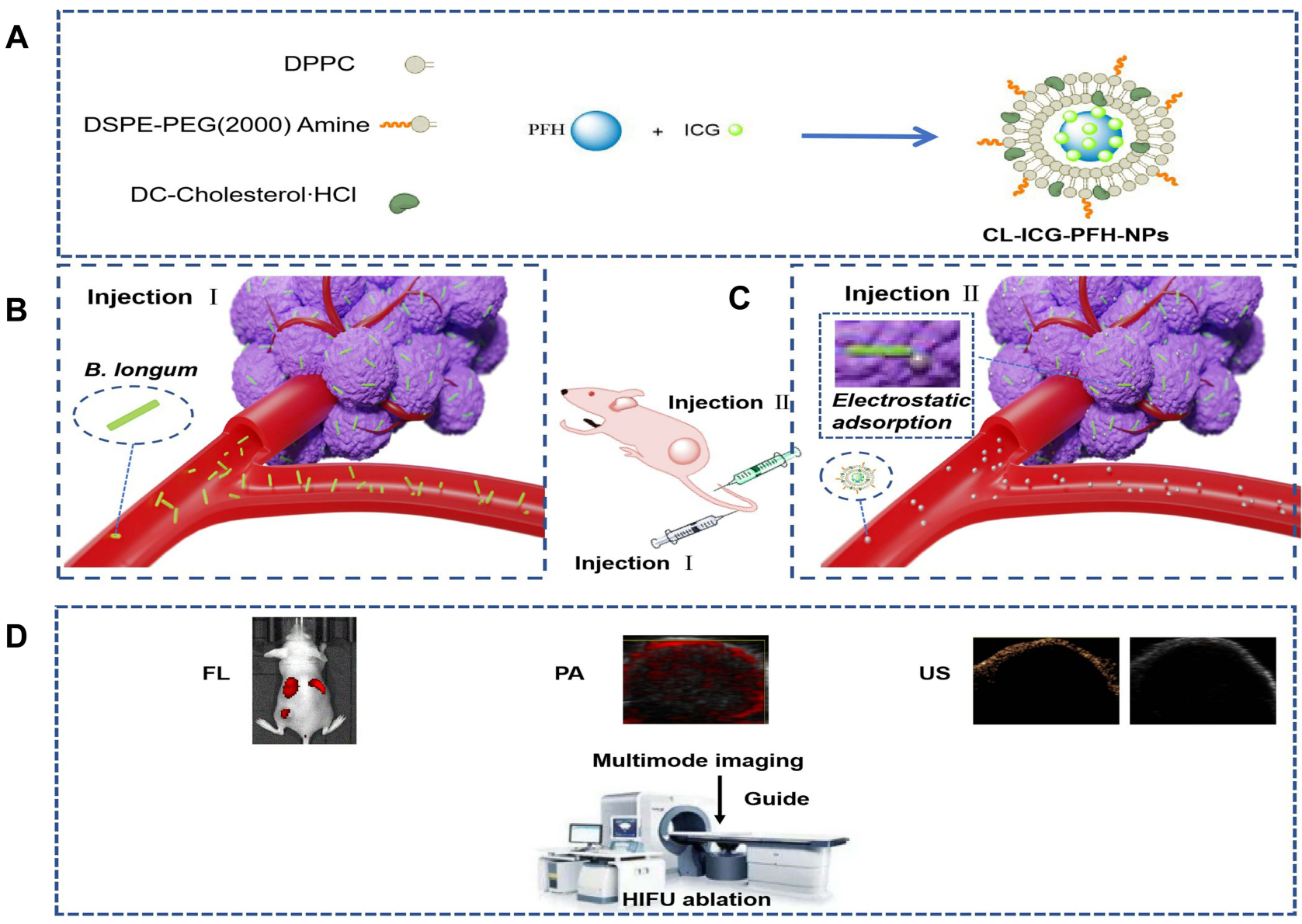

Figure I Schematic illustration of the biological targeting synergistic agent. (A) Synthetic procedures for CL-ICG-PFH-NPs. (B, C) Targeting the tumor tissue. (D) Multimode imaging and HIFU ablation.

Abbreviations: ICG, indocyanine green; PFH, perfluorohexanes; CL-ICG-PFH-NPs, ICG and PFH coloaded cationic lipid nanoparticles; HIFU, high-intensity focused ultrasound.

6-indolecarbamidinedihyrochloride (DAPI), 1.1'dioctadecy3,3,3'-tetramethylindocarbocyaninepe

rcholrate (DiI), fluorescein isothiocyanate (FITC) was purchased from Beyotime Biotech Ltd, Co. Perfluorohexanes ( $\mathrm{PFH}$, a PFC compound with a boiling point of $56^{\circ} \mathrm{C}$ ) were purchased from Biofroxx Ltd, Co. (Shanghai, China).

All chemicals were analytical grade and used without further purification.

\section{Methods}

Preparation of ICG and PFH Coloaded Cationic Lipid Nanoparticles (CL-ICG-PFH-NPs)

The cationic lipid nanoparticles encapsulating ICG and PFH were prepared by a two-step emulsion method. ${ }^{40}$ First, a mixture of $5 \mathrm{mg}$ of DPPC, $2 \mathrm{mg}$ of DSPE-PEG (2000)-amine and $2 \mathrm{mg}$ of DC-cholesterol was dissolved into $10 \mathrm{~mL}$ of $\mathrm{CHCL}_{3}$, and the solution was moved to the rotary evaporator (Yarong Inc., Shanghai, China) at $52^{\circ} \mathrm{C}$ to remove the organic solvent and form the lipid films. One hour later, $2 \mathrm{~mL}$ of deionized (DI) water was added to hydrate the lipid films for further use. Next, a mixture of $0.2 \mathrm{~mL}$ of ICG aqueous solution $(5 \mathrm{mg} / \mathrm{mL})$ and $0.2 \mathrm{~mL}$ of $\mathrm{PFH}$ was sonicated in an ice bath using a sonicator (Sonics and Materials, Inc., USA) with $100 \mathrm{~W}$ of power for $2 \mathrm{~min}$ (5 $\mathrm{s}$ on and $5 \mathrm{~s}$ off) as the first-step emulsion. After that, the ICG-PFH mixture was added to the lipid films and emulsified with a power of $150 \mathrm{~W}$ for another $5 \mathrm{~min}(5$ $\mathrm{s}$ on and $5 \mathrm{~s}$ off). Finally, the ICG and PFH coloaded cationic lipid nanoparticles were purified by centrifugation (8000 rpm, $5 \mathrm{~min}$ ) three times and stored at $4^{\circ} \mathrm{C}$ for further use. To prepare fluorescent nanodroplets, the fluorescent dye DiI $(1 \mathrm{mg})$ was added to the lipid solution, and aluminum foil was used to prevent light exposure.

\section{Bifidobacterium longum Culture}

Bifidobacterium longum (ATCC 15707) was cultured in MRS broth for $24 \mathrm{~h}$ under anaerobic conditions at $37^{\circ}$ 
C. B. longum was purified by centrifugation $(1000 \mathrm{rpm}, 10$ $\min , 4^{\circ} \mathrm{C}$ ). The $B$. longum that had been harvested were resuspended and diluted with phosphate-buffered saline (PBS, $\mathrm{pH} 7.4$ ), and the concentration of B. longum was adjusted to approximately $2 \times 10^{8}$ bacilli $/ \mathrm{mL}$.

\section{Characterization of CL-ICG-PFH-NPs and B. longum}

The morphologic characterization of CL-ICG-PFH-NPs was observed by transmission electron microscopy (TEM) (Hitachi 7500, Hitachi Ltd., Tokyo, Japan) and optical microscopy (OM, Olympus CKX41; Canada). The particle size distribution and zeta potential of CL-ICG-PFH-NPs and B. longum were measured by a Malvern Zetasizer Nano ZS (Malvern Instruments, UK). The stability of particle size distribution and zeta potential was also tested. The Gram stain of Bifidobacterial longum was observed by optical microscopy. The specific absorption peak of ICG was 780 $\mathrm{nm}$, and the calibration curve of ICG was obtained by measuring the absorbance of ICG at $780 \mathrm{~nm}$ with different concentrations. The loading content of ICG in the cationic lipid nanoparticles was determined by measuring the unbound concentration of ICG in the supernatant with a UV spectrophotometer (NanoDrop 2000, Thermo Scientific, USA) at an absorbance wavelength of $780 \mathrm{~nm}$. The loading efficiency and loading content were calculated by the following equation: loading efficiency $(\%)=($ total ICG-unbound ICG $) /$ total ICG; loading content $(\%)=($ total ICG-unbound ICG)/total lipid nanoparticles.

\section{Construction of the Biological Targeting Synergist}

To construct the biological targeting synergist, the CL-ICG -PFH-NPs $(1 \mathrm{mg} / \mathrm{mL})$ and Bifidobacterial longum $\left(1 \times 10^{6}\right.$ bacilli/mL) were mixed in a volume ratio of $3: 1$, and the mixed solution reacted at room temperature for $5 \mathrm{~min}$.

To observe the connection between CL-ICG-PFH-NPs and Bifidobacterial longum, confocal dishes were divided into two groups: the noncationic group (Lip-ICG-PFH$\mathrm{NPs}+$ B. longum) and the cationic group (CL-ICG-PFHNPs + B. longum $)$. FITC-labeled B. longum $\left(1 \times 10^{6}\right.$ bacilli/ $\mathrm{mL}$ ) was added to both groups, DiI-labeled Lip-ICG-PFHNPs $(1 \mathrm{mg} / \mathrm{mL})$ were added to the noncationic group, and DiI-labeled CL-ICG-PFH-NPs $(1 \mathrm{mg} / \mathrm{mL})$ were added to the cationic group. All the dishes were wrapped in aluminum foil to avoid light exposure and sent for confocal laser scanning microscopy (CLSM) (Nikon A1, Japan).

To detect the binding efficiencies of CL-ICG-PFH-NPs and Bifidobacterial longum, flow cytometry (FCM) was used. After preparing the DiI-labeled CL-ICG-PFH-NPs
$(1 \mathrm{mg} / \mathrm{mL})$ and Lip-ICG-PFH-NPs $(1 \mathrm{mg} / \mathrm{mL})$, the specimens were divided into three groups: $B$. longum separately group (Control), noncationic group (Lip-ICG-PFH-NPs+ B. longum) and cationic group (CL-ICG-PFH-NPs + B. longum), B. longum $\left(1 \times 10^{6}\right.$ bacilli $\left./ \mathrm{mL}\right)$ were added to every group.

\section{Photothermal Properties Detection}

To examine the photothermal effects, $0.1 \mathrm{~mL}$ of CL-ICGPFH-NPs containing ICG at concentrations of 10 to $25 \mu \mathrm{g}$ / $\mathrm{mL}$ were added to 96 -well plates and irradiated with an $808 \mathrm{~nm}$ laser at a power intensity of $1.5 \mathrm{~W} / \mathrm{cm}^{2}$ for $5 \mathrm{~min}$. Samples with the same ICG concentration were treated with different power intensities from 0.75 to $2 \mathrm{~W} / \mathrm{cm}^{2}$ for $5 \mathrm{~min}$. The temperature changes were recorded by an infrared thermal imaging camera (Fotric 226, Shanghai, China).

The effects of increased temperature on the CL-ICGPFH-NPs phase transition were assessed by optical microscopy.

\section{In vitro $\mathrm{FL}, \mathrm{PA}$ and US Imaging}

The in vitro FL imaging was performed on the Xenogen IVIS Spectrum imaging system (Perkin Elmer, USA) using a $745 \mathrm{~nm}$ excitation wavelength and an $800 \mathrm{~nm}$ filter. Then, $0.2 \mathrm{~mL}$ of sample solutions differing in their concentration of ICG from 1 to $6 \mu \mathrm{g} / \mathrm{mL}$ was added to $96-$ well plates. The FL average value was obtained using the Xenogen IVIS Spectrum imaging system.

PA imaging experiments were performed on the Vevo LASER PA imaging system (VisualSonics, Inc., Toronto, Canada) with the following parameters: frequency: 21 MHz; PA gain: $52 \mathrm{~dB}$; 2D gain: $18 \mathrm{~dB}$; wavelength: 810 $\mathrm{nm}$. Sample solutions of $0.2 \mathrm{~mL}$ differing in their concentration of ICG from 10 to $60 \mu \mathrm{g} / \mathrm{mL}$ were added to an agar gel phantom for PA imaging. The PA average value was obtained using a Vevo LAZR PA imaging system.

To evaluate the US imaging ability of the nanoparticles, PBS, CL-PFH-NPs, and CL-ICG-PFH-NPs were each placed into an agar gel phantom and irradiated with an 808 $\mathrm{nm}$ laser at a power intensity of $2.0 \mathrm{~W} / \mathrm{cm}^{2}$ for $5 \mathrm{~min}$. US imaging before and after laser irradiation was conducted on ultrasonic diagnostic imaging equipment (Mylab 90, Esaote, Italy) in conventional B mode and contrast mode. The mean echo intensity within the region of interest (ROI) was measured using DFY software (Institute of Ultrasound Imaging, Chongqing Medical Sciences).

All measurements were repeated three times. 


\section{Animals and Model Establishment}

All animal studies were conducted under a protocol approved by the Animal Ethics Committee of Chongqing Medical University. Every operation in this work was conducted with the Guidelines for the Care and Use of Laboratory Animals. For the establishment of the tumor model, each nude mouse (female BALB/c nude mice 20 g, 4-6 weeks old) was subcutaneously injected with $1 \times 10^{6}$ MDA-MB-231 cells suspended in $100 \mu \mathrm{L}$ of PBS solution into the left thigh muscle. Multimodal imaging and HIFU ablation were performed when the tumor grew to about 10 $\pm 0.5 \mathrm{~mm}$ in diameter.

\section{Targeted Detection}

Five tumor-bearing mice were randomly chosen, and then intravenously injected with $200 \mu \mathrm{L}$ of B. longum $\left(1 \times 10^{6}\right.$ bacilli/mL). After 7 days, all tumor-bearing mice were euthanized, the tumor and major organs of the tumorbearing mice were harvested, and a part of the tissues was fixed with $4 \%$ paraformaldehyde for Gram staining.

To further examine the tumor-targeting ability of the biological targeting synergistic agent, 30 tumor-bearing mice were randomly divided into 2 groups: group 1: PBS + CL-ICG-PFH-NPs, and group 2: B. longum + CL-ICGPFH-NPs; $n=15$ for each group. The mice in group 1 were injected intravenously with $200 \mu \mathrm{L}$ of PBS, and the mice in group 2 were injected intravenously with $200 \mu \mathrm{L}$ of B. longum $\left(1 \times 10^{6}\right.$ bacilli $\left./ \mathrm{mL}\right)$. After 7 days, the mice in groups 1 and 2 were injected intravenously with $200 \mu \mathrm{L}$ of DiI-labeled CL-ICG-PFH-NPs. At predetermined time intervals after injection of the nanoparticles for $(24 \mathrm{~h}, 48$ $\mathrm{h}$ and $72 \mathrm{~h}$ ), 5 mice were randomly selected from each of the two groups and sacrificed. The tumors of the mice were extracted and cut into ultrathin sections $(6 \mu \mathrm{m})$, and the CL-ICG-PFH-NPs in the tumor tissues were observed by CLSM.

\section{In vivo $F L, P A$ and $U S$ Imaging}

For in vivo FL imaging, mice were randomly allocated to three groups: group 1: B. longum, group 2: CL-ICG-PFHNPs, and group 3: B. longum +CL-ICG-PFH-NPs; $\mathrm{n}=5$ per group. The mice in groups 1 and 3 were injected intravenously with $200 \mu \mathrm{L}$ of $B$. longum $\left(1 \times 10^{6}\right.$ bacilli $\left./ \mathrm{mL}\right)$, and the mice in group 2 were injected intravenously with $200 \mu \mathrm{L}$ of PBS. After 7 days, the mice in groups 2 and 3 were injected intravenously with $200 \mu \mathrm{L}$ of CL-ICG-PFH-NPs $(1 \mathrm{mg} / \mathrm{mL})$, and the mice in group 1 were injected intravenously with 200 $\mu \mathrm{L}$ of PBS. Images were acquired at 6 h, 24 h, 48 h, and 72 $\mathrm{h}$ after the second injection.

For in vivo PA and US imaging, mice were randomly allocated to four groups: group 1: PBS, group 2: B. longum, group 3: CL-ICG-PFH-NPs, group 4: B. longum +CL-ICG-PFH-NPs; $\mathrm{n}=5$ per group. The mice in groups 2 and 4 were injected intravenously with $200 \mu \mathrm{L}$ of $B$. longum $\left(1 \times 10^{6}\right.$ bacilli $\left./ \mathrm{mL}\right)$, and the mice in groups 1 and 3 were injected intravenously with $200 \mu \mathrm{L}$ of PBS. After 7 days, the mice in groups 3 and 4 were injected intravenously with $200 \mu \mathrm{L}$ of CL-ICG-PFH-NPs (1 mg/ $\mathrm{mL}$ ), and the mice in groups 1 and 2 were injected intravenously with $200 \mu \mathrm{L}$ of PBS. PA images were obtained at $0 \mathrm{~h}, 6 \mathrm{~h}, 24 \mathrm{~h}, 48 \mathrm{~h}$, and $72 \mathrm{~h}$ after the second injection. Forty-eight hours after the second injection, the mice in all groups were irradiated with an $808 \mathrm{~nm}$ laser at a power intensity of $2.0 \mathrm{~W} / \mathrm{cm}^{2}$ for $5 \mathrm{~min}$. US imaging before and after laser irradiation was conducted on ultrasonic diagnostic imaging equipment in conventional $\mathrm{B}$ mode and contrast mode.

\section{In vivo HIFU Ablation Therapy}

A Model-JC200 Focused Ultrasound Tumor Therapeutic system (Chongqing Haifu Medical Technology Co., Ltd., Chongqing, China) was used for all HIFU experiments. The focal length, diameter, and operating frequency were 100-250 mm, 100-300 mm, and 0.5-2 MHz, respectively. A high-energy US beam was emitted from the therapeutic transducer, while changes in the targeted tissue were monitored by the diagnostic transducer in real-time.

Twenty tumor-bearing mice were randomly divided into four groups: group 1: $\mathrm{PBS}+\mathrm{HIFU}$, group 2: B. longum+ HIFU, group 3: CL-ICG-PFH-NPs+ HIFU, group 4: B. longum + CL-ICG-PFH-NPS $+\mathrm{HIFU;} \mathrm{n}=5$ per group. The mice in groups 2 and 4 were injected intravenously with $200 \mu \mathrm{L}$ of $B$. longum $\left(1 \times 10^{6}\right.$ bacilli $\left./ \mathrm{mL}\right)$, and the mice in groups 1 and 3 were injected intravenously with $200 \mu \mathrm{L}$ of PBS. After 7 days, the mice in groups 3 and 4 were injected intravenously with $200 \mu \mathrm{L}$ of CL-ICG-PFH-NPs $(1 \mathrm{mg} / \mathrm{mL})$, and the mice in groups 1 and 2 were injected intravenously with $200 \mu \mathrm{L}$ of PBS. The mice were placed in the prone position on the HIFU treatment bed, and the tumor site was completely immersed in degassed water. All mice were treated with the same power for systematic evaluation by HIFU ablation experiments (acoustic power $150 \mathrm{~W}$, irradiation duration $3 \mathrm{~s}$ ). ${ }^{38}$ During HIFU treatment, the tumor tissues of ablation appeared as a strong echo in the HIFU images. Before and after ablation, the grayscale of the targeted area 
was automatically compared by the Gray Val 1.0 software affiliated with the HIFU device. At $24 \mathrm{~h}$ after HIFU treatment, the mice were sacrificed, and the tumors were harvested. The tumor was cut into the $1-2 \mathrm{~mm}$ section series along the axis of the sonic beam. Then, the tissue was stained with $1 \%$ TTC solution in a $37^{\circ} \mathrm{C}$ water bath for $30 \mathrm{~min}$. The coagulative necrosis volume (V) and energy efficiency factor (EEF) were calculated according to the following formulas: $\mathrm{V}\left(\mathrm{mm}^{3}\right)=(\pi / 6) \times$ length $\times$ width $\times$ depth and $\operatorname{EEF}\left(\mathrm{J} / \mathrm{mm}^{3}\right)=$ $\eta \mathrm{Pt} / \mathrm{V}$, where $\eta$ is the focus coefficient of the HIFU transducer ( $\eta$ was set to 0.7 in the instrument), P(W) is the total acoustic power of the HIFU, and $t(s)$ is the total treatment time. EEF represents the ultrasonic energy required to ablate a unit volume of a tumor or other lesion. The remaining coagulation necrosis area and adjacent tumor tissues were placed in $4 \%$ paraformaldehyde for HE staining to observe the degree of tumor necrosis. To evaluate apoptosis, the terminaldeoxynucleotidyl transferase-mediated nick end labeling (TUNEL) method was performed. Immunohistochemical staining with antibodies against proliferating cell nuclear antigen (PCNA) was performed to assess tumor cell proliferation.

\section{Biocompatibility Test}

To examine the toxicity of CL-ICG-PFH-NPs, human umbilical vein endothelial cells (HUVECs, The HUVEC cells were purchased commercially from the Chinese Academy of Sciences Cell Bank) were seeded in a 96-well plate at a density of 5000 cells per well for $24 \mathrm{~h}$. Then, 100 $\mu \mathrm{L}$ of CL-ICG-PFH-NPs at different concentrations were added followed by incubation for another $24 \mathrm{~h}$. Cell viability was determined by CCK- 8 assay.

To evaluate the biosecurity of the biological targeting synergistic agent, blood samples were collected 3, 7 and 14 days after injection of the biological targeting synergistic agent for biochemical examinations of complete blood count (WBC, white blood cell; RBC, red blood cell; HGB, hemoglobin; PLT, platelets), liver functional markers (ALT, alanine aminotransferase; TP, total protein; ALB, albumin; AST, aspartate transaminase; AKLP, alkaline phosphatase; TBIL, total bilirubin), a kidney functional marker (CREA, creatinine; BUN, blood urea nitrogen) and myocardial enzyme spectrum (LDH, lactate dehydrogenase).

\section{Statistical Analysis}

All data were analyzed with GraphPad Prism version 6.0. Quantitative data were expressed as the mean \pm standard deviation. Student's $t$-test was utilized to determine statistical significance for two groups. One-way analysis of variance (ANOVA) was used for multiple-group comparisons. $P$ values $<0.05$ were considered statistically significant (NS, no significance, $\quad * P<0.05, \quad * * P<0.01, \quad * * * P<0.001$, $* * * * P<0.0001)$.

\section{Results and Discussion Synthesis and Characterization of CL-ICG-PFH-NPs and B. longum}

CL-ICG-PFH-NPs were synthesized via a two-step emulsion method to simultaneously encapsulate hydrophilic ICG and inert PFH as the core of the nanoparticles (Figure 2A).

The uniform lipid film included DPPC, DSPE-PEG (2000) and DC-CHOL. The initial PFH/ICG emulsion was obtained through the sonication and mixing of PFH and ICG, which was temporarily stable and needed to be further stabilized by the prepared lipid film through the second emulsification approach, forming the ICG and PFH coloaded cationic lipid nanoparticles (CL-ICG-PFHNPs). The optical microscopy and TEM images of CLICG-PFH-NPs are shown in Figure 2B and C. It has been demonstrated that CL-ICG-PFH-NPs are generally spherical in shape with good monodispersity. The mean diameter of the CL-ICG-PFH-NPs was $318.8 \pm 17.3 \mathrm{~nm}$ (PDI=0.147) (Figure 2E), which can effectively penetrate the capillary endothelial tumor gap. ${ }^{41}$ PEGylation of the lipid nanoparticles can overcome the key problem of rapid elimination by the mononuclear phagocytic system (MPS) in the system cycle and increase the passive accumulation by prolonging the circulation of the nanoparticles, thus minimizing the side effects of the nanoreagent. ${ }^{42,43}$ The average zeta potentials of CL-ICG-PFH-NPs and B. Longum were $28.4 \pm 4.71 \mathrm{mV}$ (Figure $2 \mathrm{~F}$ ) and $-29.7 \pm 3.31 \mathrm{mV}$ (Figure 2G), respectively, which indicates that they could be connected by electrostatic adsorption. Furthermore, to assess the stability of CL-ICG-PFH-NPs, the size and the zeta potential changes over 9 days were tested. There were no significant changes in either size (Figure $2 \mathrm{H}$ ) and zeta potential (Figure 2I), the results indicated that the CL-ICG -PFH-NPs has good stability. According to the standard curve of ICG aqueous solution (Figure 2J), the ICG loading efficiency and loading content of CL-ICG-PFH-NPs were approximately $74 \%$ and $9.09 \mathrm{wt} \%$, respectively, which were much higher than that of the LIP-ICG reported previously because the double emulsification method can 
A
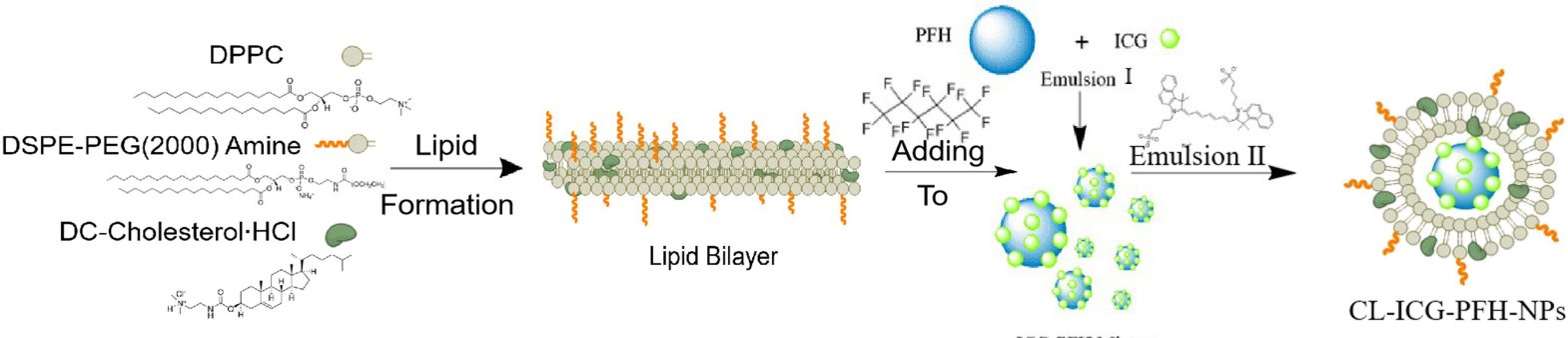

ICG-PFH Mixture

B

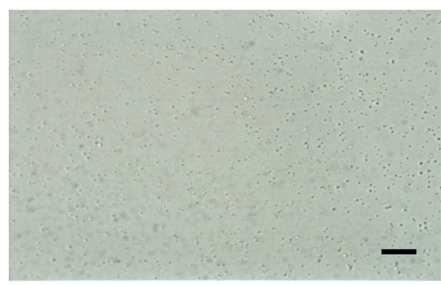

E

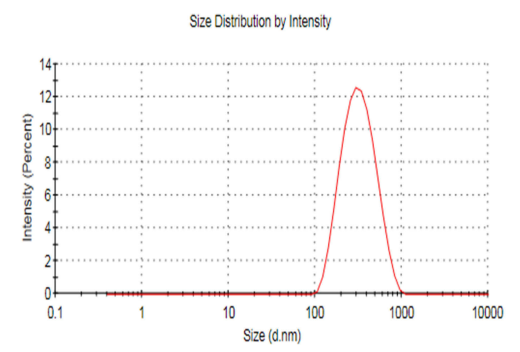

H

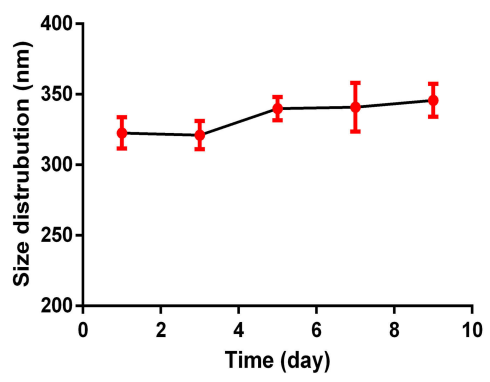

C

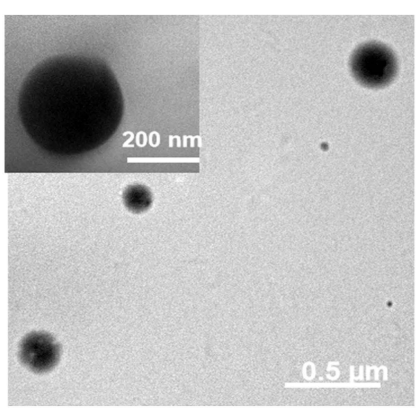

$\mathbf{F}$

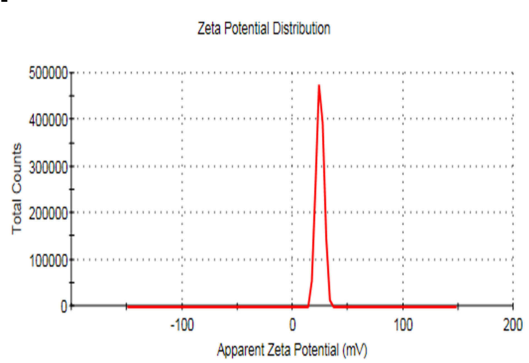

I

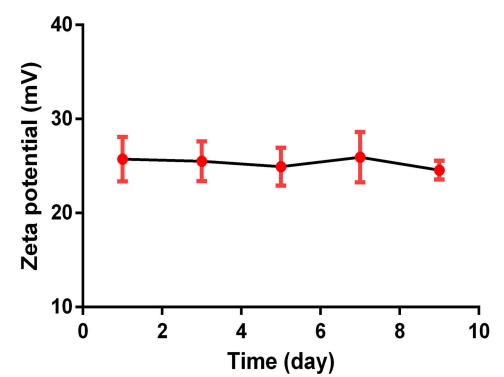

D

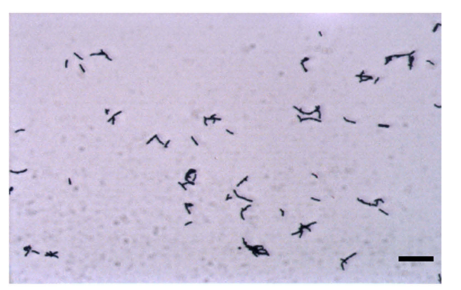

G

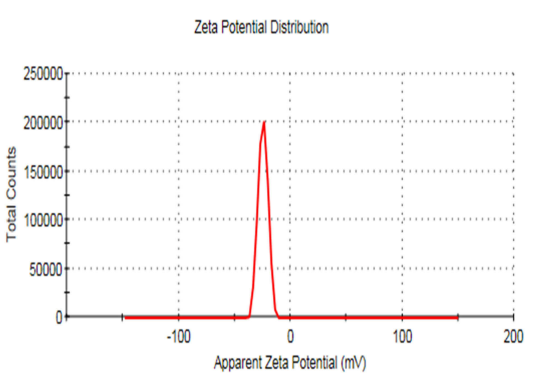

J

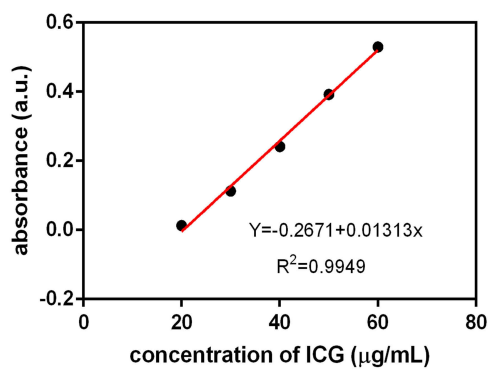

Figure 2 Synthesis and characterization of CL-ICG-PFH-NPs and B. longum. (A) Synthetic procedures for CL-ICG-PFH-NPs via two-step emulsion method.(B) Optical microscope image of CL-ICG-PFH-NPs (400 x magnification), the scale bar is $10 \mu \mathrm{m}$. (C) TEM images of CL-ICG-PFH-NPs. (D) Optical microscope image of Gram stain of bifidobacteria longum ATCC 15707 (400 × magnification), the scale bar is $10 \mu \mathrm{m}$. (E, F) Particle size distribution and surface zeta potential of CL-ICG-PFH-NPs. (G) Surface zeta potential of B. longum. $(\mathbf{H})$ The particle size and (I) zeta potential of CL-ICG-PFH-NPs over a period of 9 days in deionized water. (J) The calibration curve of ICG obtained from the absorbance at $780 \mathrm{~nm}$ with different concentrations of ICG.

Abbreviations: ICG, indocyanine green; PFH, perfluorohexanes; CL-ICG-PFH-NPs, ICG and PFH coloaded cationic lipid nanoparticles; HIFU, high-intensity focused ultrasound; TEM, transmission electron microscopy.

greatly improve the encapsulation efficiency of watersoluble drugs. ${ }^{44,45}$

\section{In vitro Construction of the Biological Targeting Synergist}

The surface of the CL-ICG-PFH-NPs prepared in this study had a positive potential, and the surface of
B. Longum had a negative potential. Therefore, CL-ICGPFH-NPs could adhere to the surface of the negatively charged B. Longum by electrostatic adsorption. DiIlabeled red fluorescent CL-ICG-PFH-NPs and Lip-ICGPFH-NPs could be observed by CLSM with excitation and emission wavelengths of $549 \mathrm{~nm}$ and $565 \mathrm{~nm}$, respectively, and FITC-labeled green fluorescent B. longum could be 
seen with excitation and emission wavelengths of $494 \mathrm{~nm}$ and $518 \mathrm{~nm}$, respectively. As shown in Figure 3A, there was a significantly stronger red fluorescent signal on the surface of B. longum in the cationic group compared with the noncationic group. It was indicated that more CL-ICGPFH-NPs adhered to the surface of B. longum. To detect the binding efficiencies of CL-ICG-PFH-NPs and
B. longum, flow cytometry (FCM) was used. As shown in Figure $3 \mathrm{~B}$, the connection rate in the cationic group was $98.67 \%$, while that of the noncationic group was only $3.73 \%$. All the above results indicate the successful and effective construction of biological targeting synergists, and this phenomenon lays a foundation for the role of B. longum retaining CL-ICG-PFH-NPs in tumor tissues.

A

FITC

Dil

Merge
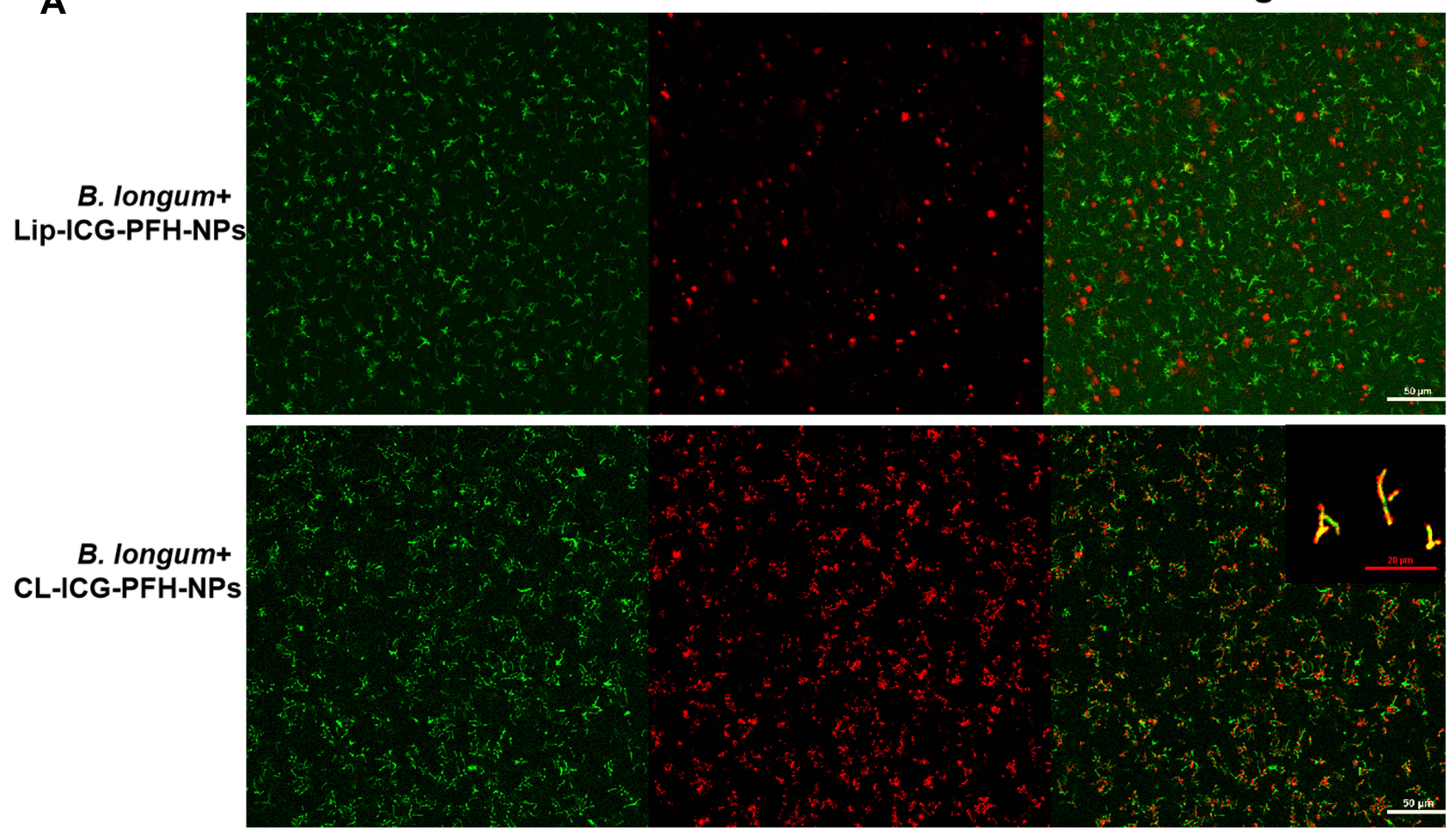

B

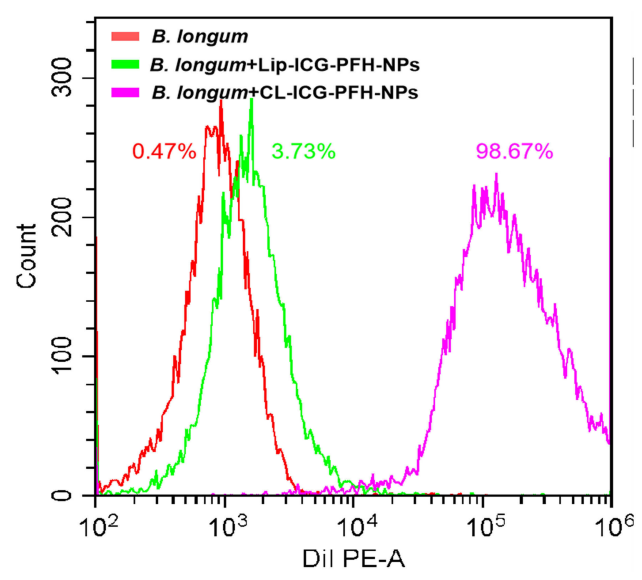

Figure 3 In vitro construction of the biological targeting synergist. (A) CLSM images of Dil-labeled NPs and CL-ICG-PFH-NPs (red), FITC-labeled B. longum (green). Compared with the noncationic group, there was a significantly stronger red fluorescent signal on the surface of $B$. longum in the cationic group, the scale bar is $50 \mu m$. (B) FCM image of binding efficiencies of CL-ICG-PFH-NPs and B. longum.

Abbreviations: CLSM, confocal laser scanning microscopy; Dil, I.I'dioctadecy-3,3,3'-tetramethylindocarbocyaninepercholrate; FITC, fluorescein isothiocyanate; DAPI, 2-(4-amidinophenyl)-6-indolecarbamidinedihyrochloride; FCM, flow cytometry; ICG, indocyanine green; PFH, perfluorohexanes. 


\section{In vitro Photothermal Evaluation}

The photothermal capacity of CL-ICG-PFH-NPs was assessed in an aqueous dispersion. When the power density of the NIR laser increased, the temperature of the aqueous dispersion of CL-ICG-PFH-NPs also increased (Figure 4A and $\mathrm{B}$ ), which followed a power-dependent manner at a fixed ICG concentration of CL-ICG-PFH-NPs. As shown in Figure $4 \mathrm{C}$, under the same laser irradiation, the temperature of CL-ICG-PFH-NP dispersions with different ICG concentrations rapidly increased compared with PBS, demonstrating the excellent photothermal effect of CL-ICG-PFH-NPs.

Images of CL-ICG-PFH-NPs before and after irradiation were observed by optical microscopy. After irradiation, the nanoparticles became larger and fused into microbubbles (Figure 4D). Since the ICG in the CL-ICG-
PFH-NPs is stimulated by the laser, heat is generated, the temperature rises, the PFH in the nanoparticles is heated and liquid-gas phase transition occurs, so the nanoparticles become larger and fuse into microbubbles.

These results indicated that the CL-ICG-PFH-NPs have excellent photothermal conversion capability, which lays a foundation for the subsequent imaging experiments.

\section{In vitro $\mathrm{FL}, \mathrm{PA}$ and US Imaging}

The FL, PA and US imaging properties of CL-ICG-PFHNPs were evaluated. ICG is an NIR-absorbing fluorescent contrast agent. ${ }^{46}$ To test the FL capability, the fluorescence of CL-ICG-PFH-NPs with different ICG concentrations was examined in a well plate. As shown in Figure 5A, the fluorescence signal generated by CL-ICG-PFH-NPs
A

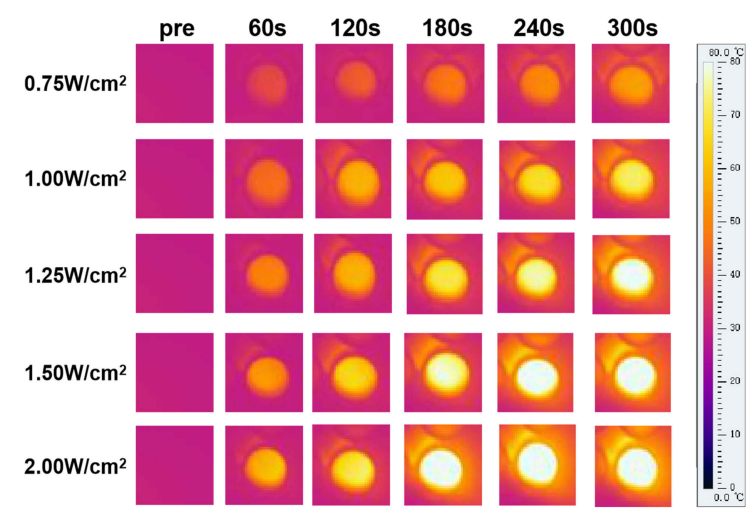

C

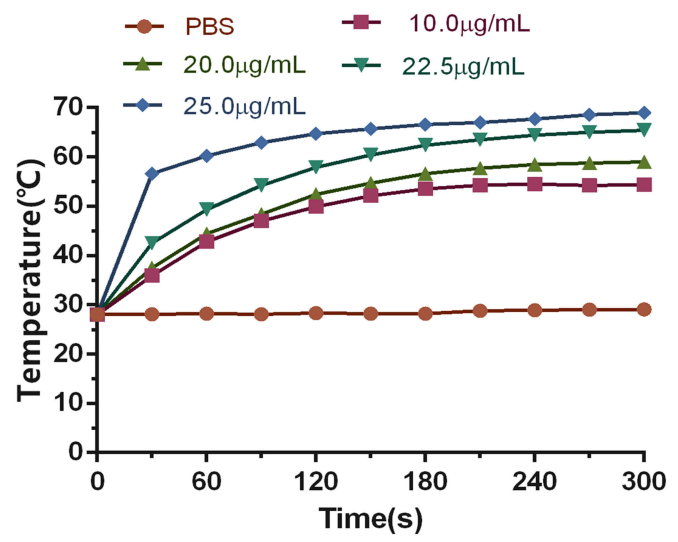

B

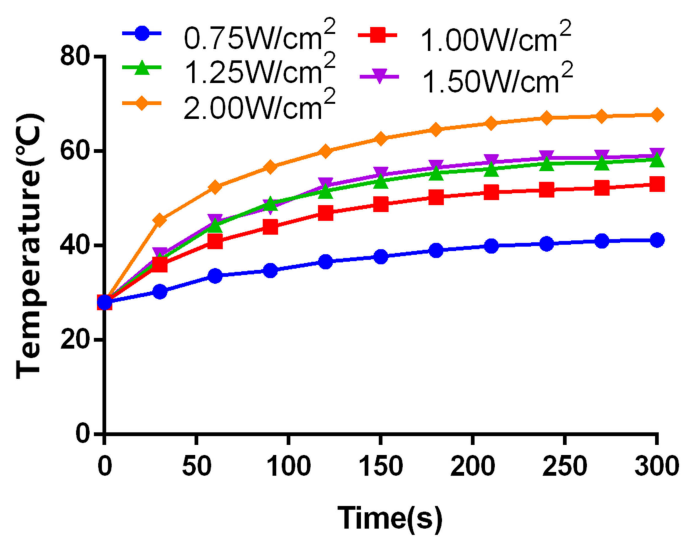

D

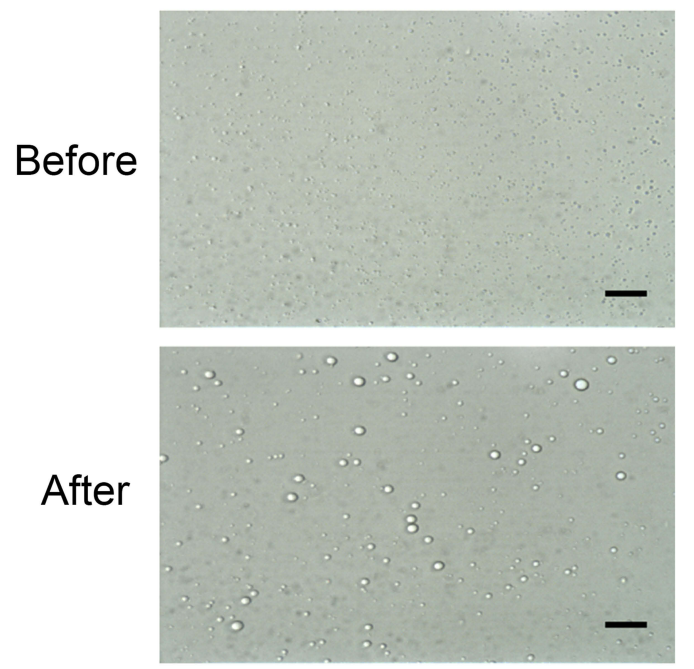

Figure 4 In vitro photothermal evaluation. (A) The picture of temperature evolution of CL-ICG-PFH-NPs under different power density laser. (B) Temperature evolution curves over time corresponding to different power density laser $(n=3)$. (C) Temperature evolution curves over time corresponding to different concentration $\mathrm{CL}-\mathrm{ICG}$-PFHNPs $(n=3)$. (D) Optical microscope image of CL-ICG-PFH-NPs before and after laser irradiation ( $400 \times$ magnification). After irradiation, the nanoparticles became larger and fused into microbubbles, the scale bar is $10 \mu \mathrm{m}$.

Abbreviations: ICG, indocyanine green; PFH, perfluorohexanes. 
A

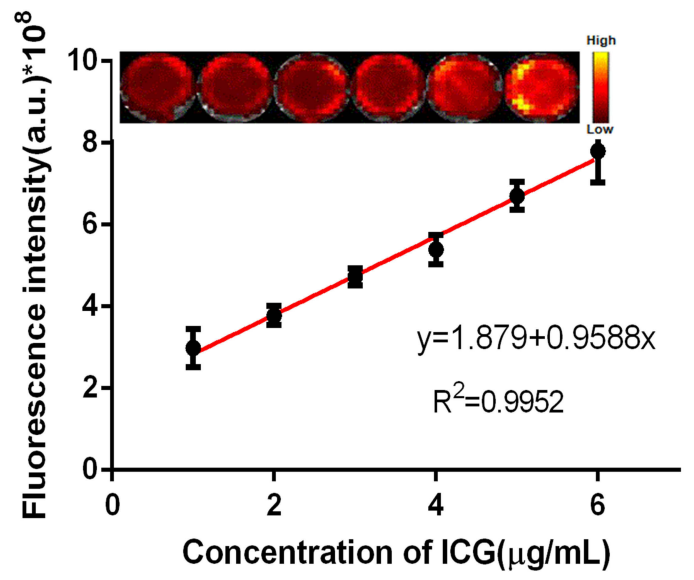

C

\section{CL-ICG-PFH-NPS}
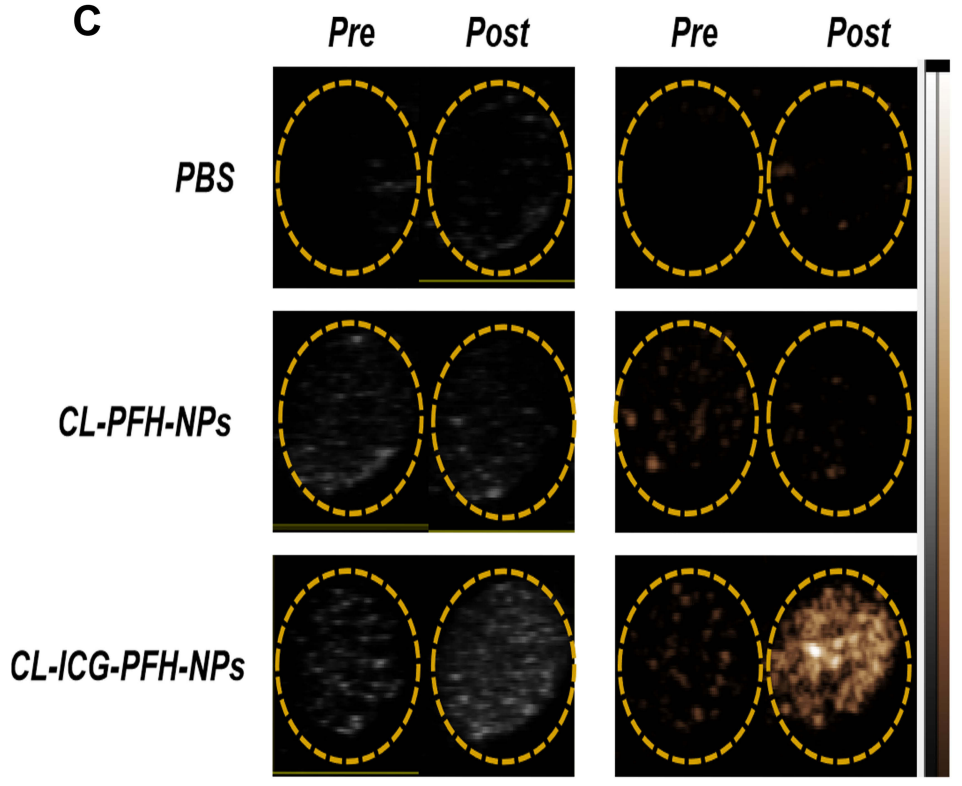

B

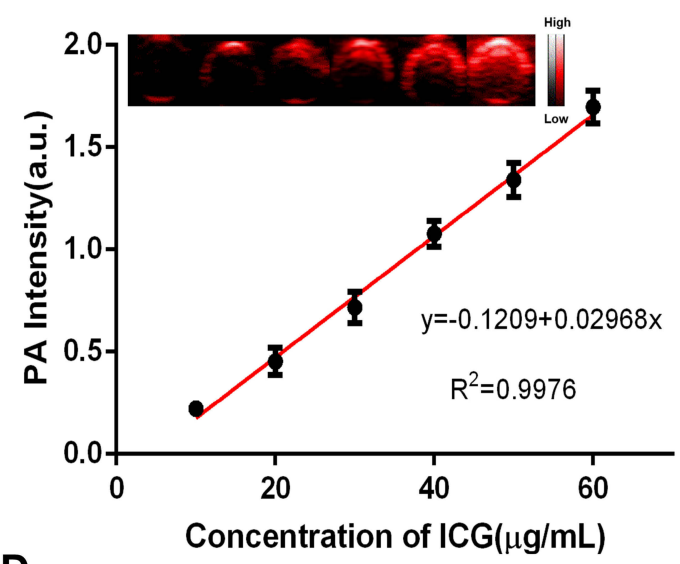

D

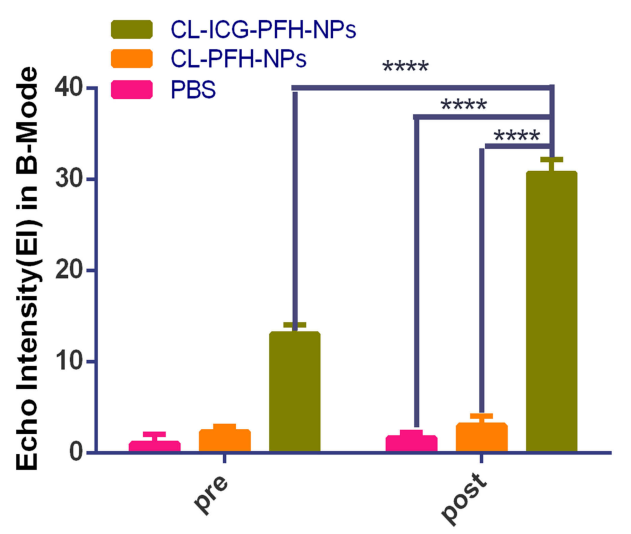

E

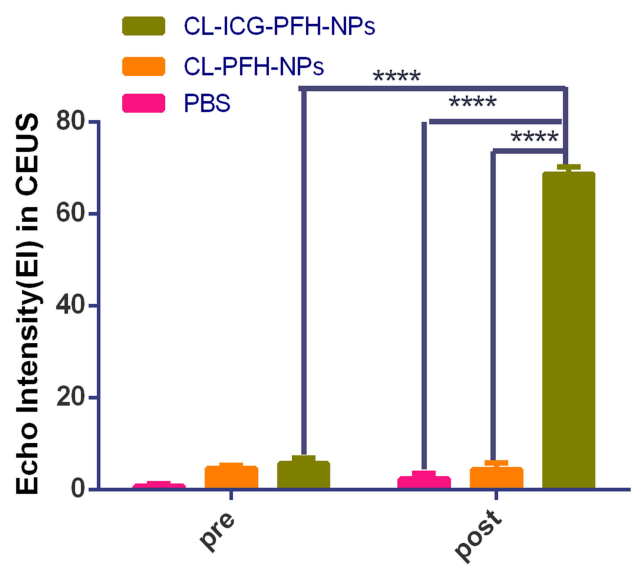

Figure 5 In vitro FL, PA and US imaging. (A) FL intensities and FL images of CL-ICG-PFH-NPs with different concentrations of ICG ( $n=3$ ). (B) PA signals and PA images of CL-ICG-PFH-NPs with different concentrations of ICG $(n=3)$. (C) Ultrasound images of CL-ICG-PFH-NPs before and after laser irradiation in vitro. (D) El in B-mode and (E) El in CEUS imaging before and after irradiation in different groups $(n=3)$. There was a significant increase in the $B$-mode imaging and CEUS imaging effects in the CL-ICGPFH-NP group when compared with the other two groups after irradiation $(* * * * P<0.0001)$. The difference was also detected in the $C L-I C G-P F H-N P$ group before and after irradiation $(* * * * P<0.0001)$.

Abbreviations: ICG, indocyanine green; PFH, perfluorohexanes; FL, fluorescence; PA, photoacoustic; US, ultrasound; El, echo intensity; B-mode, brightness modulation; CEUS, contrast-enhanced ultrasound.

strengthened linearly. The CL-ICG-PFH-NPs had strong absorption in the NIR region, and it was obvious that the PA signal was linearly enhanced with ICG concentration under the excitation wavelength of $810 \mathrm{~nm}$, making it an excellent PA contrast agent (Figure 5B).
The results of US imaging (B-mode and CEUS) and semiquantitative echo intensities (EI) in the PBS, CL-PFHNPs, CL-ICG-PFH-NP groups with or without irradiation are shown in Figure 5C-E. In the CL-ICG-PFH-NP group, the EI of B-mode (Figure 5D) increased by 3.2 times and was 
over 10-fold higher than that of the other two groups after irradiation. Moreover, the EI of CEUS (Figure 5E) increased by 10.12-fold and was 13.8-fold stronger than that of the other two groups after irradiation. There was a significant increase in the B-mode imaging and CEUS imaging effects in the CL-ICG-PFH-NP group when compared with the other two groups after irradiation $(* * * * P<0.0001)$. The difference was also detected in the CL-ICG-PFH-NP group before and after irradiation $(* * * * P<0.0001)$. The above results further prove the photothermal effects of ICG. Only when the nanoparticles are encapsulated with ICG and PFH can the nanoparticles be excited by the laser to produce the liquid-gas phase change and realize US imaging.

Targeting Ability of the Biological Targeting Synergist in vivo

Gram staining of the tissue sections showed that abundant B. longum was detected in tumors (Figure 6A), indicating that B. longum actively targets tumors and then
A

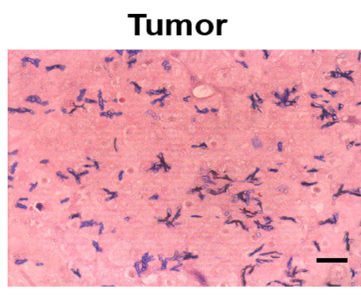

Spleen

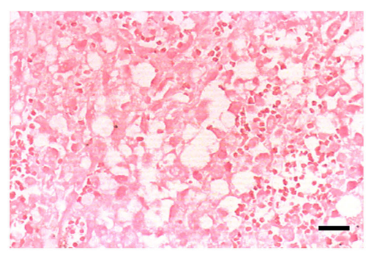

Heart

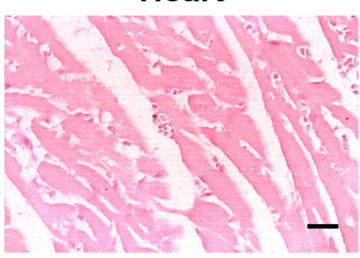

Lung

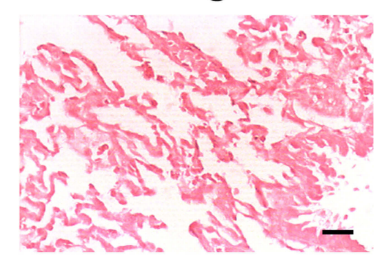

Liver

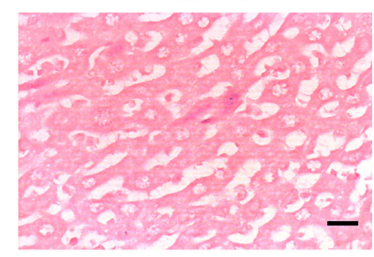

Kidney

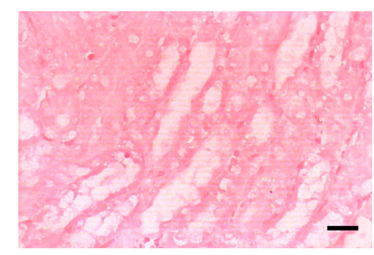

B

B. longum + CL-ICG-PFH-NPs

\section{CL-ICG-PFH-NPs}

\section{DAPI}
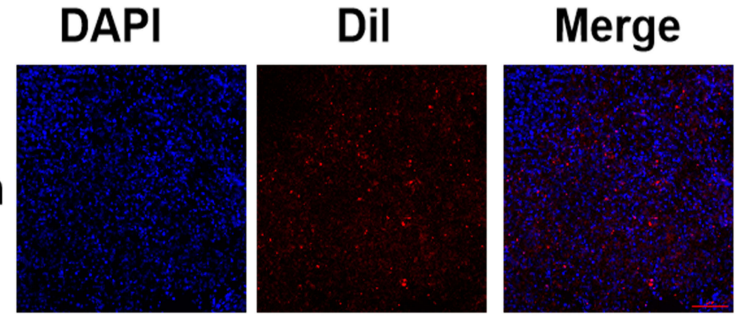

48h
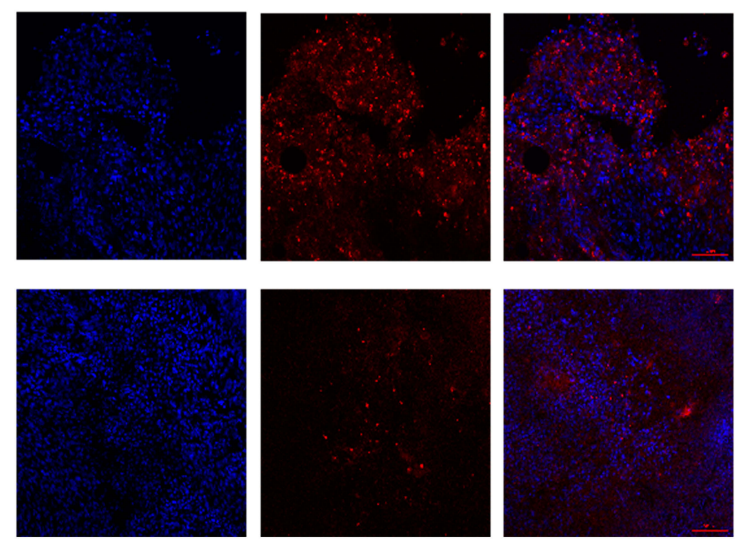

$72 \mathrm{~h}$

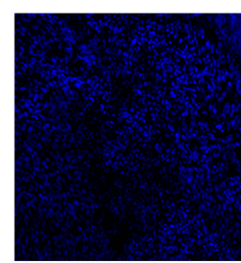

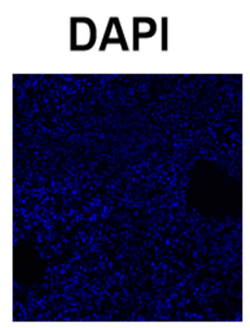

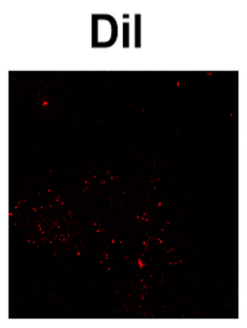

Merge
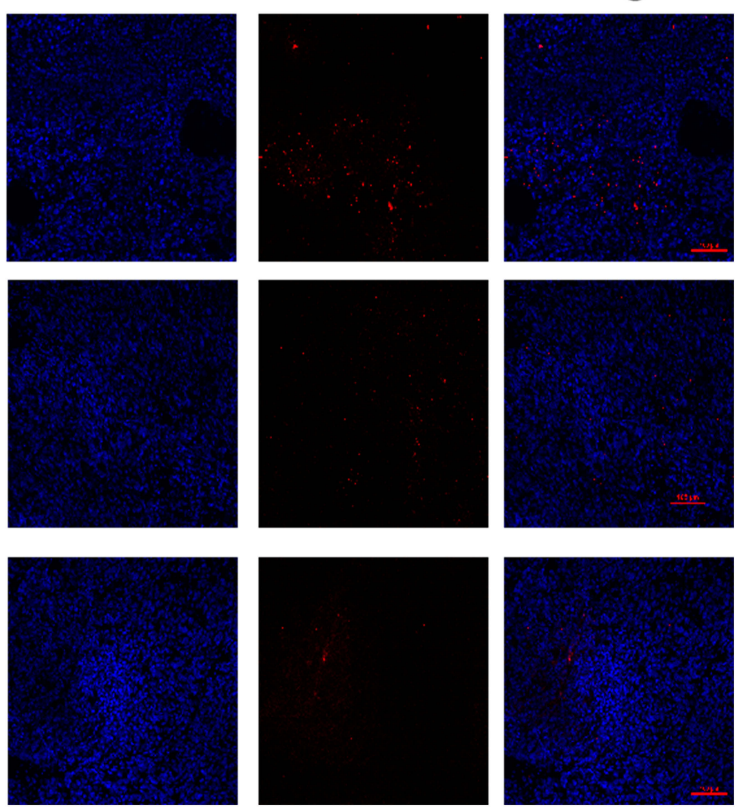

Figure 6 Targeting ability of the biological targeting synergist in vivo. (A) Gram staining for evaluating tumor targeting of $B$. longum (400 $\times$ magnification), the scale bar is 10 $\mu \mathrm{m}$. (B) CLSM images of tumor tissue at different points $(24,48,72 \mathrm{~h}$ ) after injection of Dil-labeled CL-ICG-PFH-NPs (red) in the tail vein, DAPI (blue) marks nuclei, the scale bar is $100 \mu \mathrm{m}$.

Abbreviations: CLSM, confocal laser scanning microscopy; Dil, I.I'dioctadecy-3,3,3'-tetramethylindocarbocyaninepercholrate; ICG, indocyanine green; PFH, perfluorohexanes. 
continuously replicated and cloned itself. In contrast, no blue-violet stained B. longum was found in the other organs.

To further prove the targeting ability of the biological targeting synergists to tumor tissues, the aggregation of DiIlabeled red fluorescent CL-ICG-PFH-NPs in tumors was observed by CLSM, and the nucleus was stained with DAPI to observe blue fluorescence. As shown in Figure $6 \mathrm{~B}$, there was a significantly stronger red fluorescence signal in group 2 (B. longum + CL-ICG-PFH-NPs) compared with group 1 (PBS+CL-ICG-PFH-NPs), and this fluorescence reached its maximum at $48 \mathrm{~h}$. The mechanism underlying this phenomenon could be complicated and may be associated with the following factors. First, because of the growth of solid tumors, a large number of anaerobic areas appear in tumors due to ischemia and hypoxia. The facultative anaerobic properties of B. longum make it easy to concentrate and multiply in the anaerobic zone of the tumor after entering the blood, indicating tumor targeting. ${ }^{47}$ Second, B. longum in the tumors can act as an anchor to urge CLICG-PFH-NP-specific migration and deposition into the tumor zone. ${ }^{39}$ All of the above phenomena prove that the biological targeting synergist has good tumor-targeting ability, and we believe that the active-targeting ability of biological targeting synergists may contribute to the accumulation at the tumor site. This lays a foundation for the imaging and treatment of tumors.

\section{In vivo $F L, P A$ and $U S$ Imaging}

The feasibility of biological targeting synergistic multimodal imaging agents was evaluated in mice bearing MDA-MB-231 tumors after systemic administration of the biological targeting synergistic agent. We first evaluated the biodistribution of the nanoliposomes on the mice by in vivo FL imaging (Figure 7A). In the B. longum+ CL-ICG-PFH-NP group, fluorescent signals were observed in the tumor area 6-72 $\mathrm{h}$ after injection of the CL-ICG-PFH-NPs. Moreover, the fluorescence intensity and range decreased slowly after reaching a peak at $48 \mathrm{~h}$ (Figure 7A and B). There was no fluorescent signal in the B. longum group. In the CL-ICG-PFH-NP group, relatively weak fluorescence was observed in the tumor area 6-72 $\mathrm{h}$ after injection of the CL-ICG-PFH-NPs. In addition, the fluorescence intensity and range rapidly decreased after reaching a peak at $24 \mathrm{~h}$ (Figure $7 \mathrm{~A}$ and $\mathrm{B}$ ). The fluorescence intensity of the tumor tissue in the B. longum+CL-ICG-PFHNP group was greater than that in the CL-ICG-PFH-NP group throughout the observation period $(* * P<0.01$; $* * * P<0.001$; Figure 7A and B). In particular, B. longum + CL-ICG-PFH-NP group exhibited relatively longer retention in the tumor region. To confirm tumor accumulation, tumor-bearing mice were sacrificed $48 \mathrm{~h}$ postinjection, and the major organs were collected for fluorescent imaging. Ex vivo imaging (Figure 7C) and the corresponding quantitative results (Figure 7C) showed that both groups exhibited high signals in the liver and spleen, owing to the uptake of nanoparticles by the reticuloendothelial system, which is abundant in the spleen and liver. Moreover, there was a significant difference between the two groups of nanoliposomes that accumulated in the tumor region $(* * * P<0.001)$, further confirming the targeting capacity of our biological targeting synergistic agent to tumor.

Then, the in vivo PA imaging efficacy of biological targeting synergistic was examined using an $810 \mathrm{~nm}$ laser as the excitation light source. After intravenous injection, the cross-sectional PA images of the mice were obtained at different time intervals. As shown in Figure 7D and E, there was no PA signal in the PBS and B. longum groups. A strong PA signal was observed in the tumor area 48 $\mathrm{h}$ after the injection in the B. longum+CL-ICG-PFH-NPs group, which was significantly different from the CL-ICGPFH-NP group. The PA intensity of the tumor tissue in the B. longum+CL-ICG-PFH-NP group was greater than that in the CL-ICG-PFH-NP group $(* * * * P<0.0001)$ throughout the observation period (Figure 7E). Both FL and PA imaging techniques revealed that $B$. longum increased the deposition amount and residence time of CL-ICG-PFHNPs in tumor tissues.

The US imaging ability of the biological targeting synergistic was evaluated by using an $808 \mathrm{~nm}$ laser as an irradiation light source. Forty-eight hours after the injection, the US imaging (B-mode and CEUS) and semiquantitative echo intensities (EI) increased significantly in the B. longum +CLICG-PFH-NP group after laser irradiation at $808 \mathrm{~nm}$ $(* * * * P<0.0001)$. There were no significant US values in the other groups (Figure $7 \mathrm{~F}-\mathrm{H}$ ). This phenomenon may be related to the following factors. First, during laser irradiation, ICG could strongly absorb the laser energy to create heat, resulting in the liquid-gas phase transition of $\mathrm{PFH}$. At the same time, the ultrasonic pressure wave generated by the thermal expansion of tissue could enhance the acoustic signal, resulting in an US signal increase. ${ }^{48}$ Second, B. longum in tumor tissue increases the deposition and retention duration of CL-ICG-PFH-NPs.

These similar results all confirmed the fact that biological targeting synergistic had the highest accumulation at $48 \mathrm{~h}$ postinjection and significantly prolonged the blood 


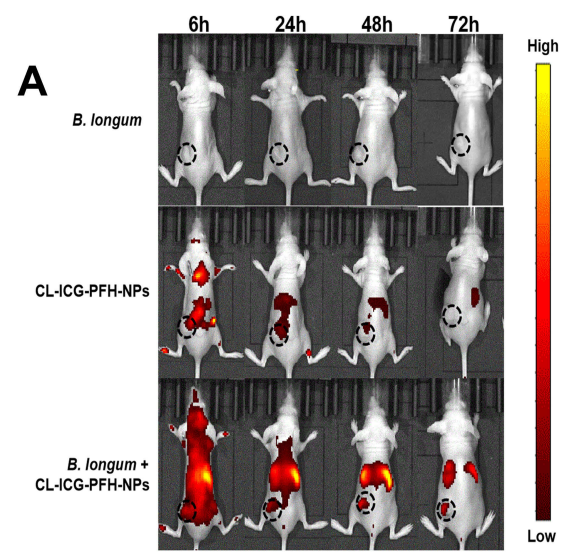

C
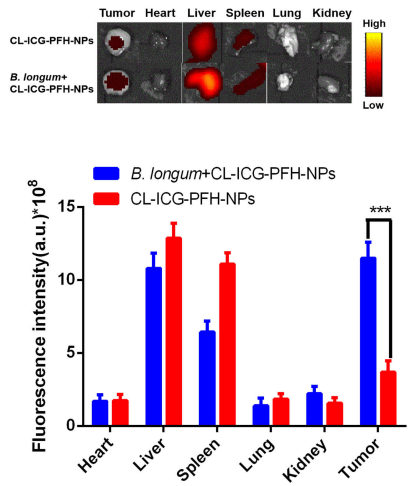

E

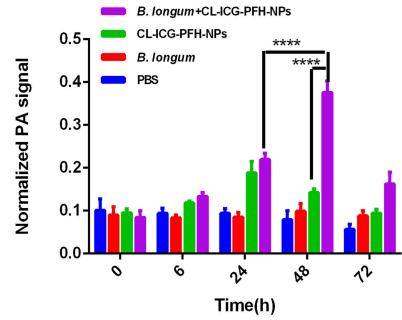

G

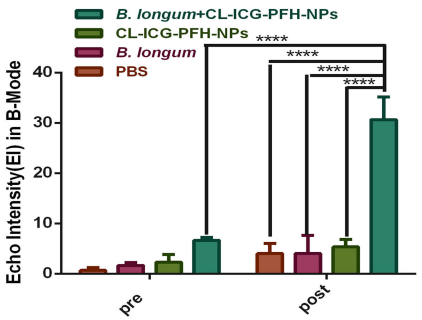

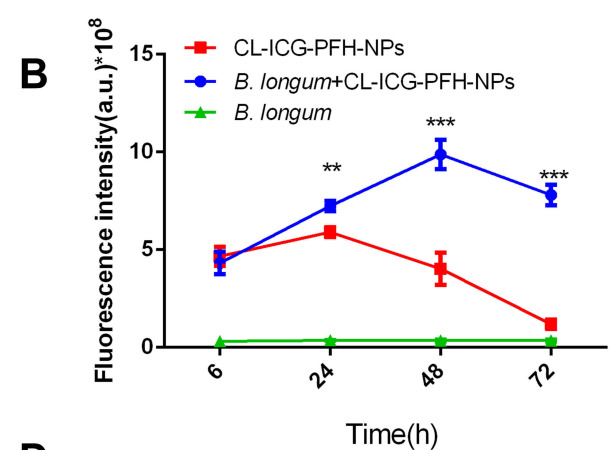

D

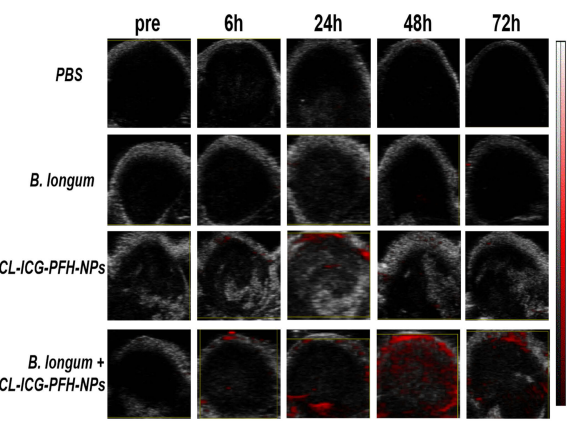

F

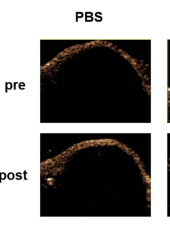

B. longum

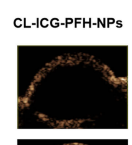

B. longum+
CL-ICG-PFH-NPs

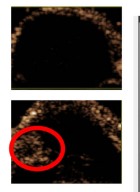

PBS
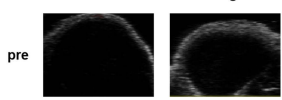

post
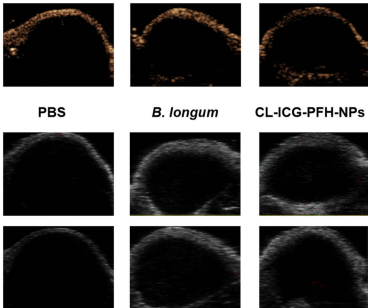

CL-ICG-PFH-NPS

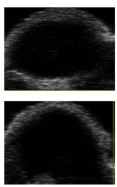

B. longum+
CL-ICG-PFH-NPs
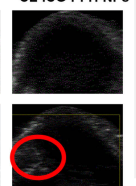

H

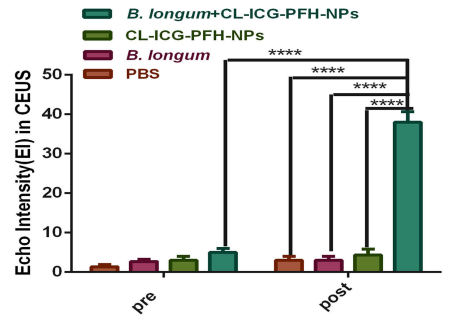

Figure 7 In vivo FL, PA and US imaging. (A) Fluorescence images of tumor-bearing mice at different time points (6, 24, 48, 72 h) after injection of PBS and CL-ICG-PFH-NPs in the B. longum group, CL-ICG-PFH-NP group and B. longum + CL-ICG-PFH-NPs group, the black dotted circle marks the tumor nodules. (B) Quantitative fluorescence in the intensity of tumor site, there was a significant increase of the fluorescence intensity in the $B$. longum $+C L-I C G-P F H-N P$ group when compared with the other two groups after injection. ( $n=5$, $* * P<0.01$, $\left.{ }^{* * *} \mathrm{P}<0.00 \mathrm{I}\right)$. (C) Ex vivo fluorescence images of major organs and tumors dissected from mice $48 \mathrm{~h}$ post-injection in CL-ICG-PFH-NP group and $B$. longum $+\mathrm{CL}-\mathrm{ICG}-\mathrm{PFH}-\mathrm{NPs}$ group. Quantitative fluorescence in the intensity of major organs and tumors, there was a significant difference in the fluorescence intensity in the $B$. longum + CL-ICG-PFH-NP group when compared with the CL-ICG-PFH-NP group $(n=5, * * * P<0.00 I)$. (D) PA images of tumor-bearing mice at different time points $(0,6,24,48,72 \mathrm{~h})$ after injection of PBS and CL-ICGPFH-NPs in the PBS group, $B$ longum group, CL-ICG-PFH-NP group and B. longum + CL-ICG-PFH-NPs group. (E) The corresponding PA signals at the tumor sites of tumor-bearing mice after injection of PBS and CL-ICG-PFH-NPs in the PBS group, $B$ longum group, CL-ICG-PFH-NP group and B. longum + CL-ICG-PFH-NP group at different time points. There was a significant increase of the PA signals in the $B$. longum $+\mathrm{CL}-\mathrm{ICG}-\mathrm{PFH}-\mathrm{NP}$ group when compared with the other three groups after injection $48 \mathrm{~h}$. The difference was also detected in the B. longum + CL-ICG-PFH-NP group at $48 \mathrm{~h}$ and $24 \mathrm{~h}$. ( $\mathrm{n}=5$, $* * * * P<0.000 \mathrm{I})$. (F) Ultrasound images of tumor-bearing mice before and after laser irradiation $48 \mathrm{~h}$ after PBS and CL-ICG-PFHNPs injection in the PBS group, $B$ longum group, CL-ICG-PFH-NP group and B. longum + CL-ICG-PFH-NP group. The red circle marks the area where the intensity is sigificantly enhanced. (G) El in B-mode and (H) El in CEUS imaging before and after irradiation in different groups. There was a significant difference in the B. longum + CL-ICG-PFH-NP group when compared with the other three groups after irradiation, the difference was also detected in the $B$. longum $+C L-I C G-P F H-N P$ group before and after irradiation $(n=5$, $* * * * P<0.000 I)$. Abbreviations: ICG, indocyanine green; PFH, perfluorohexanes; FL, fluorescence; PA, photoacoustic; US, ultrasound; El, echo intensity; B-mode, brightness modulation; CEUS, contrast-enhanced ultrasound. 
circulation time of the nanoparticles. The possible reasons are shown as follows: most nanoparticle-based therapies rely on the enhanced permeability and retention (EPR) effect to diffuse into the tumor regions and deliver a payload. The limitation is that the EPR effect alone can not guarantee a better therapeutic effect. In this experiment, the CL-ICG-PFH-NPs in biological targeting synergistic agent group would assumedly accumulate in tumor sites through the EPR effect and the recognition of $B$. longum in the tumor tissues. CL-ICG-PFH-NPs could bind to B. longum in tumors by electrostatic adsorption. Therefore, compared with the pure nanoparticle group without $B$. longum, the tumor sites of the biological targeting synergistic group enriched more nanoparticles, which had better imaging effect. FL, PA and US imaging are nonradioactive imaging with different advantages. FL imaging visualizes the dynamic distribution of nanoparticles within the whole body. PA imaging can monitor nanoparticle accumulation within the tumor site with improved imaging depth and resolution. US imaging is a clinically used diagnostic method due to its desirable features, such as its real-time monitoring capability, high safety and sensitivity, low cost, availability, and portability. Triplemodal imaging could combine the advantages of each modality well, synergistically providing essential information to accurately delineate tumor size and location, visualizing the dynamic distribution of the biological targeting synergistic and guiding HIFU therapy.

\section{In vivo Synergistic HIFU Ablation Therapy}

HIFU ablation was performed $48 \mathrm{~h}$ after injection of the nanoparticles. This time point was chosen because multimodal imaging showed that after $48 \mathrm{~h}$, the maximum concentration of nanoparticles in the tumor was observed. After HIFU irradiation, the grayscale of the tumor target region in all the groups changed to varying degrees (Figure $8 \mathrm{~A}$ and $\mathrm{B}$ ). The mean grayscale (Figure $8 \mathrm{~A}$ and $\mathrm{B}$ ) difference in the $\mathrm{B}$. longum + CL-ICG-PFH-NPs+HIFU group was significantly higher than in the other groups $(* * * P<0.001)$. We removed the tumor tissue from the nude rats to perform TTC staining and measure the ablation volumes, the coagulative necrosis area was gray white, and the normal tissue was red. A sharply demarcated region between the non-ablated tissues (red) and the necrotic (white) was observed upon gross inspection in CL-ICG-PFH-NPs + HIFU group, B. longum + CL-ICG-PFH -NPs + HIFU group (Figure 8C). The results showed that the ability of the nanoparticles to destroy malignant lesions without damaging the surrounding normal tissues. The specific volume of coagulative necrosis in PBS + HIFUgroup, B. longum + HIFU group, CL-ICG-PFH-NPs + HIFU group, $B$. longum + CL-ICG-PFH-NPs + HIFU group are as follows: (9.152 \pm 2.081$), \quad(21.308 \pm 3.271), \quad(58.639 \pm 5.884)$, $(180.158 \pm 28.316) \mathrm{mm}^{3}$. The coagulative necrosis volume of the $B$. longum+CL-ICG-PFH-NPs+HIFU group, which was significantly bigger than any other groups (Figure $8 \mathrm{C}$ and $\mathrm{D}$ ). Moreover, the detailed values of EEF in PBS + HIFUgroup, B. longum + HIFU group, CL-ICG-PFH-NPs + HIFU group, B. longum + CL-ICG-PFH-NPs + HIFU group are as follows: (35.87 \pm 8.061$), \quad(15.096 \pm 2.566), \quad(5.417 \pm 0.562), \quad(1.783$ $\pm 0.276) \mathrm{J} / \mathrm{mm}^{3}$. The EEF results showed that the $B$. longum + CL-ICG-PFH-NPs+HIFU group was significantly different from the other groups (Figure 8E). Based on the above results, CL-ICG-PFH-NPs show promise as HIFU synergistic agent. Moreover, when B. longum is combined with CL-ICG-PFHNPs, more nanoparticles can be retained in tumor tissues, further enhancing the ablation effect of HIFU. To further investigate the degree of destruction after HIFU ablation, a series of pathological examinations were undertaken. HE staining showed a clear boundary between the ablated area and the non-ablated area, and obvious coagulative necrosis characteristics were easily observed in the B. longum $+\mathrm{CL}$ ICG-PFH-NPs+HIFU group. In contrast, tumor cells in nests and exhibited good cellular morphology in the PBS group. Moreover, the proliferation and apoptosis levels in the tumors revealed by the PCNA and TUNEL assays followed the same trend. PCNA expression was visualized as brown granules in cell nuclei and observed for all the groups. PCNA was reduced in the coagulation necrosis region after HIFU ablation, while it was positive in the normal tissue. In the TUNEL assay, positive staining (brown nuclei) cells represented apoptosis cells. In the B. longum+CL-ICG-PFH-NPs+HIFU group, the expression of PCNA was significantly reduced, whereas the expression of TUNEL was obviously enhanced compared with the other groups (Figure 8F). This result indicated that under the same conditions, after HIFU irradiation, the biological targeting synergistic showed the strongest synergistic effects. The possible mechanism for this phenomenon may be related to the following factors. First, during HIFU ablation, thermal ablation is able to destroy target tissues by the localized deposition of thermal energy, which transiently raises the tissue temperature to $>60^{\circ} \mathrm{C}$ and can stimulate protein denaturation and coagulation necrosis. Second, the cavitation effect of HIFU therapy can also change the function and structure of the cell membranes to kill tumor cells. Third, CL-ICG-PFH-NPs could serve as a phase change agent and form microbubbles that can facilitate HIFU ablation by 

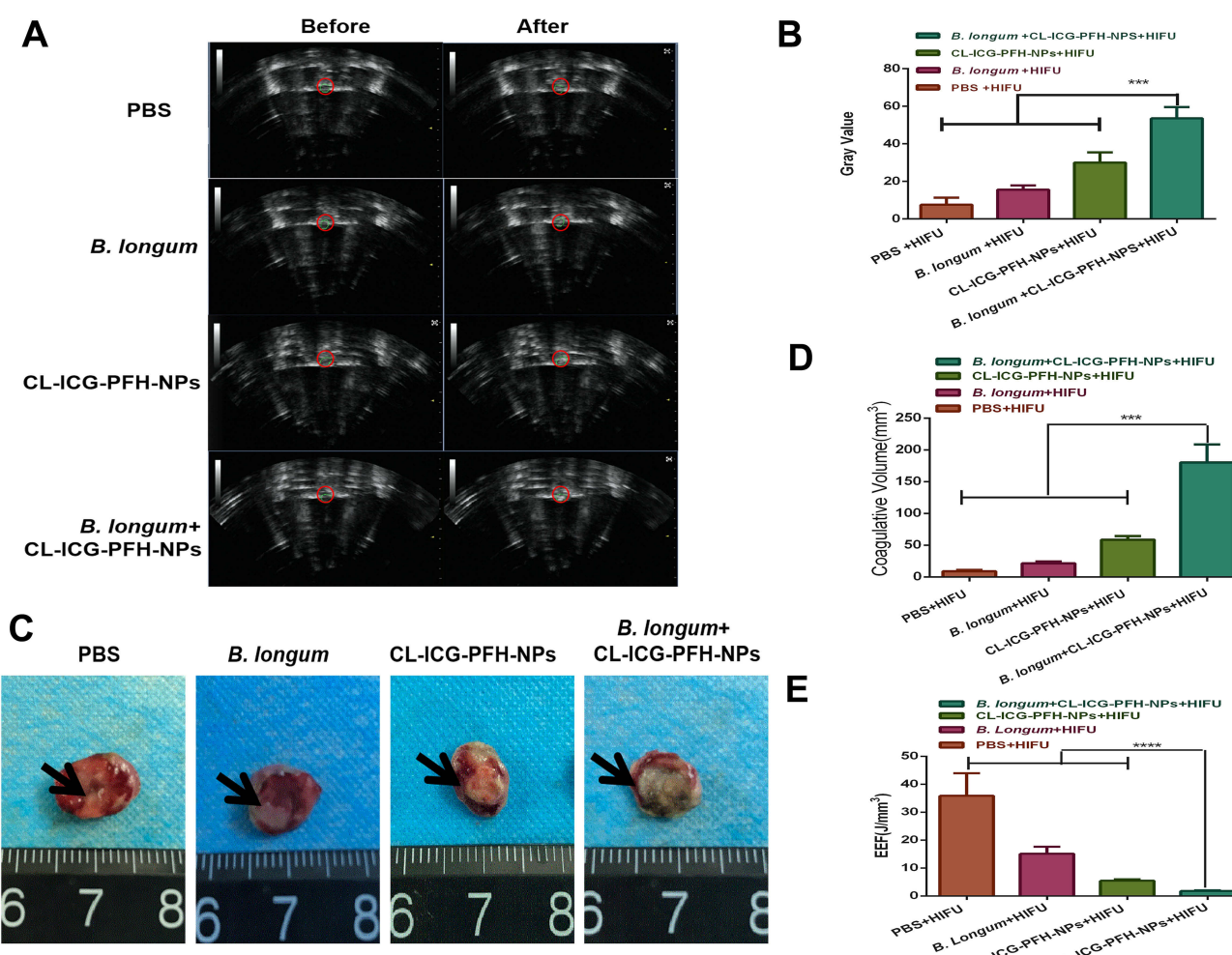

D
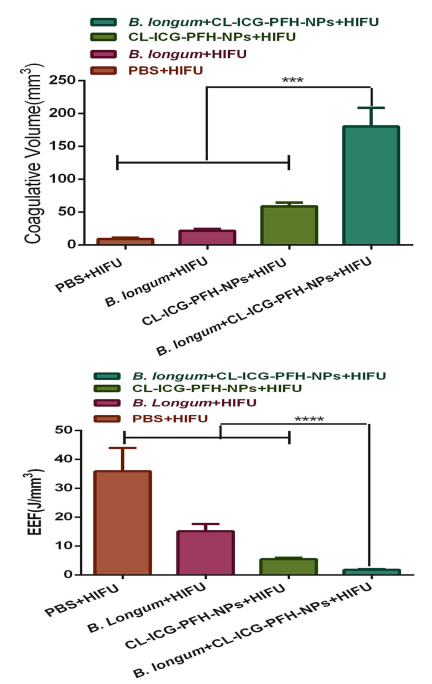

$\mathbf{F}$

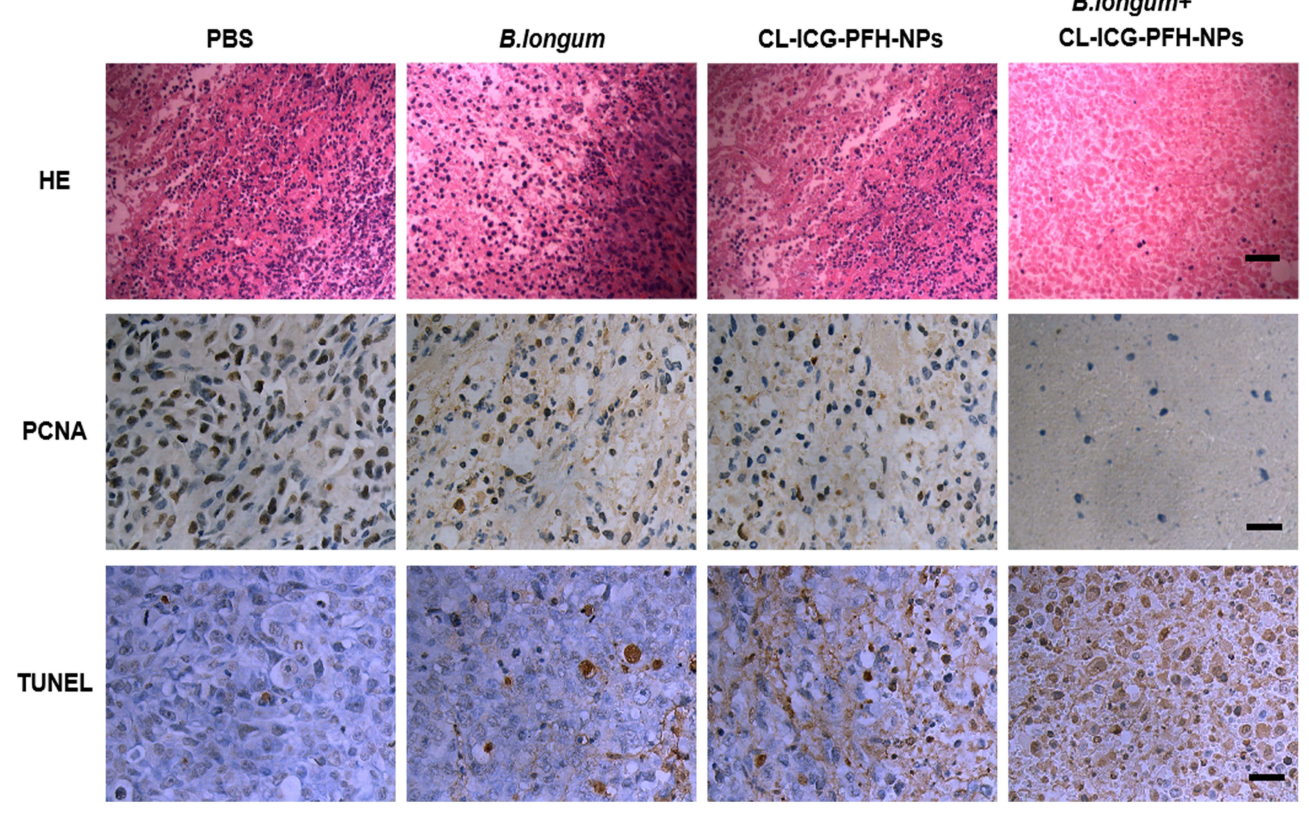

Figure 8 In vivo synergistic HIFU ablation surgery. (A) In vivo US imaging of tumor tissues (red circle) before HIFU ablation and after HIFU ablation in different group. The red dotted circle marks the tumor nodules. (B) The comparison of gray values of tumor tissues in each group after HIFU irradiation. The mean grayscale difference in the B. longum+CL-ICG-PFH$\mathrm{NPs}+\mathrm{HIFU}$ group was significantly higher than the other groups $(n=5, * * * P<0.00 \mathrm{I})$. (C) Coagulative necrosis of tumors by TTC staining after HIFU therapy, the necrotic tissue appears gray and the normal tumor tissue is red. (D) The comparison of coagulative necrosis of tumor tissues in each group after HIFU irradiation. The coagulative necrosis volume of the B. longum+CL-ICG-PFH-NPs+HIFU group was significantly bigger than any other groups $(n=5, * * * P<0.00 I)$. (E) The comparison of EEF of tumor tissues in each group after HIFU irradiation. The results showed that the $B$. longum+CL-ICG-PFH-NPs+HIFU group was significantly different from the other groups $(n=5$, $* * * * P<0.000 \mathrm{I})$. ( $\mathbf{F}$ ) Optical microscope images of tumor sections from each group after HIFU ablation were stained for HE staining, PCNA and TUNEL assay. There exists a significant boundary between the nonablated and ablated region in HE staining.( $200 \times$ magnification), the scale bar is $20 \mu \mathrm{m}$. PCNA positive cells are represented in brown and reduced in the coagulation necrosis region after HIFU ablation, TUNEL positive cells are in brown and increased in the coagulation necrosis region ( $400 \times$ magnification), the scale bar is $10 \mu \mathrm{m}$.

Abbreviations: ICG, indocyanine green; PFH, perfluorohexanes; HIFU, high-intensity focused ultrasound; HE, hematoxylin and eosin staining; PCNA, proliferating cell nuclear antigen; TUNEL, apoptosis expression; EEF, energy efficiency factor. 
mechanical effects, acoustic streaming and shear stress. Furthermore, B. longum trapped a large number of CL-ICGPFH-NPs in the tumor target area. Therefore, multimodal imaging chose the best treatment time according to the distribution of nanoparticles in the body to guide the efficient and effective treatment of tumor.

\section{Biosafety Assay}

To evaluate the cytotoxicity of CL-ICG-PFH-NPs against normal HUVECs, we used a CCK- 8 assay. The cell viabilities of HUVECs treated with CL-ICG-PFH-NPs at different concentrations were similar to the contrast groups (Figure 9A).

To evaluate the in vivo safety of biological targeting synergistic agents in the short and long term, the blood biochemical indexes in mice were examined after injection of the biological targeting synergistic. The results of the blood biochemical index showed negligible differences among all the groups under different therapeutic stages (Figure 9B and C), indicating undetectable toxicity to mice at our tested time points in both the short- and long-term.
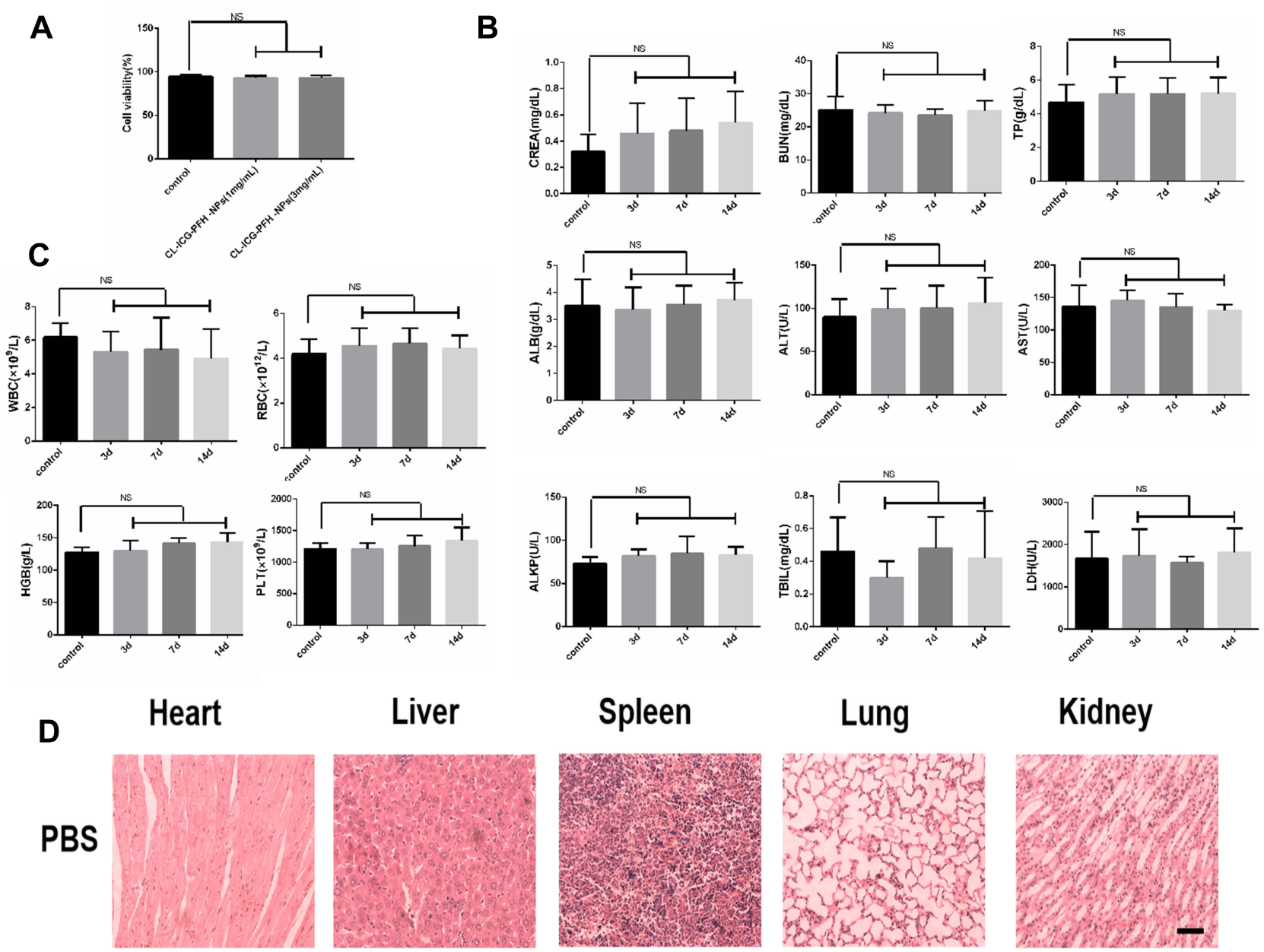

Liver
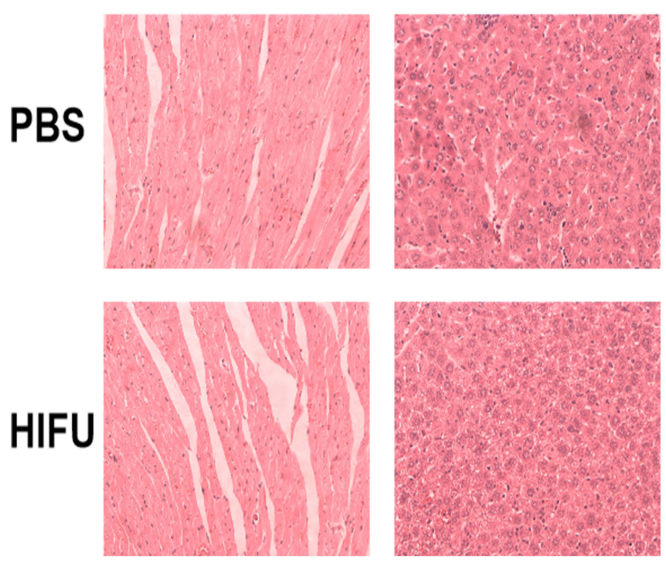

Spleen

Lung

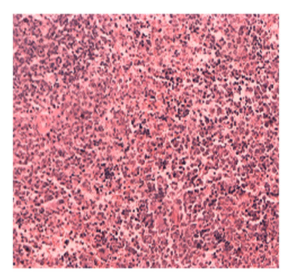

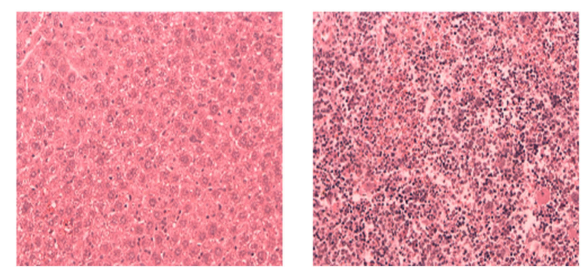

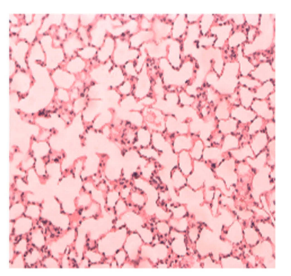

Kidney
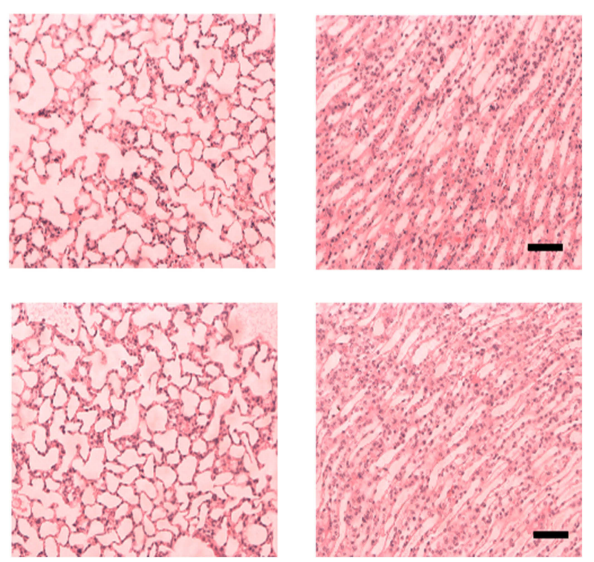

Figure 9 Biosafety assay. (A) Cell viability assay of different concentration CL-ICG-PFH-NPs incubated with HUVECs. (B) Blood biochemical indexes at different time points after biological targeting synergistic agent injections. (C) Blood routine indexes at different time points after biological targeting synergistic agent injections. (D) HE-stained images of major organs from healthy mice and mice treatment with HIFU ablation ( $200 \times$ magnification), the scale bar is $20 \mu \mathrm{m}$.

Abbreviations: HUVECS, human umbilical vein endothelial cells; WBC, white blood cell; RBC, red blood cell; HGB, hemoglobin; PLT, platelets; ALT, alanine aminotransferase; BUN, blood urea nitrogen; TP, total protein; ALB, albumin; AST, aspartate transaminase; AKLP, alkaline phosphatase; TBIL, total bilirubin; CREA, creatinine; LDH, lactate dehydrogenase; NS, no significance. 
HE staining of major organs (Figure 9D) exhibited no apparent physiological abnormalities, suggesting negligible damage to major organs after HIFU ablation with the biological targeting synergistic. All of the above results proved the biosafety of the biological targeting synergistic and tumor therapy.

\section{Conclusion}

In this paper, a biological targeting synergistic agent was successfully constructed with good stability and physicochemical properties. Both in vitro and in vivo experiments have successfully demonstrated that the biological targeting synergistic agent has good tumor tissue targeting, excellent multimodal imaging and the ability to enhance tumor treatment. The biological targeting synergistic agent can not only provide information for early diagnosis of tumors but also realize multimodal imaging, HIFU cooperation and multimodal imaging monitoring simultaneously with tumor treatment, which improves the shortcomings of HIFU treatment and has broad application prospects.

\section{Acknowledgments}

The authors are greatly thankful for the financial support by the Chongqing Research Program of Basic Research and Frontier Technology (No. cstc2019jcyj-msxmX0334).

\section{Disclosure}

The authors report no conflicts of interest in this work.

\section{References}

1. Kennedy JE. High-intensity focused ultrasound in the treatment of solid tumours. Nat Rev Cancer. 2005;5:321-327. doi:10.1038/nrc1591

2. Duc NM, Keserci B. Emerging clinical applications of high-intensity focused ultrasound. Diagn Interv Radiol. 2019;25:398-409. doi: $10.5152 / \mathrm{dir}$

3. Uchida T, Nakano M, Hongo S, et al. High-intensity focused ultrasound therapy for prostate cancer. Int J Urol. 2012;19:187-201. doi:10.1111/iju.2012.19.issue-3

4. Ning Z, Xie J, Chen Q, et al. HIFU is safe, effective, and feasible in pancreatic cancer patients: a monocentric retrospective study among 523 patients. Onco Targets Ther. 2019;12:1021-1029. doi:10.2147/ OTT.S185424>

5. Wang C. Therapeutic effects of adriamycin combined with high-intensity focused ultrasound on osteosarcoma. $J$ BUON. 2019;24:826-831.

6. Zhu B, Li J, Diao L, Ma K, Fan Y, Yang W. High-intensity focused ultrasound ablation for advanced pancreatic cancer. J Cancer Res Ther. 2019;15:831-835. doi:10.4103/jcrt.JCRT_408_18

7. Arrigoni F, Bruno F, Palumbo P, et al. Magnetic resonance-guided focused ultrasound surgery treatment of non-spinal intra-articular osteoblastoma: feasibility, safety, and outcomes in a single-center retrospective analysis. Int $J$ Hyperthermia. 2019;36:768-775. doi:10.1080/02656736.2019.1639833
8. Ter Haar G. HIFU tissue ablation: concept and devices. Adv Exp Med Biol. 2016;880:3-20.

9. Orgera G, Monfardini L, Vigna P, et al. High-intensity focused ultrasound (HIFU) in patients with solid malignancies: evaluation of feasibility, local tumour response and clinical results. Radiol Med. 2011;116:734-748. doi:10.1007/s11547-011-0634-4

10. Seok J, Woo SH, Kwon TR, et al. Role of mechanical and thermal damage in pericapsular inflammatory response to injectable silicone in a rabbit model. PLoS One. 2019;14:e0216926. doi:10.1371/journal.pone.0216926

11. Gignoux BM, Scoazec JY, Curiel L, Beziat C, Chapelon JY. High intensity focused ultrasonic destruction of hepatic parenchyma. Ann Chir. 2003;128:18-25. doi:10.1016/S0003-3944(02)00004-4

12. Zhang L, Orsi F, Arnone P, Chen W. High intensity focused ultrasound ablation: a new therapeutic option for solid tumors. J Cancer Res Ther. 2010;6:414-420. doi:10.4103/0973-1482.77064

13. Sun Y, Zheng Y, Ran H, et al. Corrigendum to "Superparamagnetic PLGA-iron oxide microcapsules for dual-modality US/MR imaging and high intensity focused US breast cancer ablation. Biomaterials. 2015;64:1. doi:10.1016/j.biomaterials.2015.06.021

14. Wu F, Wang ZB, Chen WZ, et al. Extracorporeal high intensity focused ultrasound ablation in the treatment of patients with large hepatocellular carcinoma. Ann Surg Oncol. 2004;11:1061-1069. doi:10.1245/ASO.2004.02.026

15. Li YY, Sha WH, Zhou YJ. Short and long term efficacy of high intensity focused ultrasound therapy for advanced hepatocellular carcinoma. J Gastroenterol Hepatol. 2007;22:2148-2154. doi:10.11 11/j.1440-1746.2006.04719.x

16. Blum NT, Gyorkos CM, Narowetz SJ, Mueller EN, Goodwin AP. Phospholipid-coated hydrophobic mesoporous silica nanoparticles enhance thrombectomy by high-intensity focused ultrasound with low production of embolism-inducing clot debris. ACS Appl Mater Interfaces. 2019;11:36324-36332. doi:10.1021/acsami.9b11095

17. You Y, Wang Z, Ran H, et al. Nanoparticle-enhanced synergistic HIFU ablation and transarterial chemoembolization for efficient cancer therapy. Nanoscale. 2016;8:4324 4339. doi:10.1039/C5NR08292G

18. Elhelf IAS, Albahar H, Shah U, Oto A, Cressman E, Almekkawy M. High intensity focused ultrasound: the fundamentals, clinical applications and research trends. Diagn Interv Imaging. 2018;99:349-359. doi:10.1016/j.diii.2018.03.001

19. Ektate K, Munteanu MC, Ashar H, Malayer J, Ranjan A. Chemoimmunotherapy of colon cancer with focused ultrasound and Salmonella-laden temperature sensitive liposomes (thermobots). Sci Rep. 2018;8:13062. doi:10.1038/s41598-018-30106-4

20. Chang N, Lu S, Qin D, et al. Efficient and controllable thermal ablation induced by short-pulsed HIFU sequence assisted with perfluorohexane nanodroplets. Ultrason Sonochem. 2018;45:57-64. doi:10.1016/j.ultsonch.2018.02.033

21. He K, Ran H, Su Z, Wang Z, Li M, Hao L. Perfluorohexaneencapsulated fullerene nanospheres for dual-modality US/CT imaging and synergistic high-intensity focused ultrasound ablation. Int J Nanomedicine. 2019;14:519-529. doi:10.2147/IJN

22. Lin CY, Pitt WG. Acoustic droplet vaporization in biology and medicine. Biomed Res Int. 2013;2013:404361. doi:10.1155/2013/ 404361

23. Li DS, Kripfgans OD, Fabiilli ML, Brian Fowlkes J, Bull JL. Initial nucleation site formation due to acoustic droplet vaporization. Appl Phys Lett. 2014;104:063703. doi:10.1063/1.4864110

24. Azhari H. Feasibility study of ultrasonic computed tomography-guided high-intensity focused ultrasound. Ultrasound Med Biol. 2012;38:619-625. doi:10.1016/j.ultrasmedbio.2012. 01.005

25. Napoli A, Anzidei M, Ciolina F, et al. MR-guided high-intensity focused ultrasound: current status of an emerging technology. Cardiovasc Intervent Radiol. 2013;36:1190-1203. doi:10.1007/ s00270-013-0592-4 
26. Fan HJ, Zhang C, Lei HT, et al. Ultrasound-guided high-intensity focused ultrasound in the treatment of uterine fibroids. Medicine (Baltimore). 2019;98:e14566. doi:10.1097/MD.0000000000014566

27. Qu Y, Xiao Z, Liu L, Lv F, Sheng B, Li J. Uterine peristalsis before and after Ultrasound-Guided High-Intensify Focused Ultrasound (USgHIFU) treatment for symptomatic uterine fibroids. Med Sci Monit. 2019;25:2553-2560. doi:10.12659/MSM.913392

28. Cai W, Chen X. Multimodality molecular imaging of tumor angiogenesis. J Nucl Med. 2008;49(Suppl 2):113S-28S. doi:10.2967/jnumed.107.045922

29. Kim J, Choi W, Park EY, et al. Real-time photoacoustic thermometry combined with clinical ultrasound imaging and high intensity focused ultrasound. IEEE Trans Biomed Eng. 2019. doi:10.1109/ TBME.2019.2904087

30. Liu Y, Chen S, Sun J, et al. Folate-targeted and oxygen/indocyanine green-loaded lipid nanoparticles for dual-mode imaging and photo-sonodynamic/photothermal therapy of ovarian cancer in vitro and in vivo. Mol Pharm. 2019;16:4104-4120. doi:10.1021/acs. molpharmaceut.9b00339

31. Yan F, Wu H, Liu H, et al. Molecular imaging-guided photothermal/ photodynamic therapy against tumor by iRGD-modified indocyanine green nanoparticles. $J$ Control Release. 2016;224:217-228. doi:10.1016/j.jconrel.2015.12.050

32. Yazawa K, Fujimori M, Amano J, Kano Y, Taniguchi S. Bifidobacterium longum as a delivery system for cancer gene therapy: selective localization and growth in hypoxic tumors. Cancer Gene Ther. 2000;7:269-274. doi:10.1038/sj.cgt.7700122

33. Taniguchi S, Shimatani Y, Fujimori M. Tumor-targeting therapy using gene-engineered anaerobic-nonpathogenic Bifidobacterium longum. Methods Mol Biol. 2016;1409:49-60.

34. Kizaka-kondoh S, Inoue M, Harada H, Hiraoka M. Tumor hypoxia: a target for selective cancer therapy. Cancer Sci. 2003;94:1021-1028. doi:10.1111/j.1349-7006.2003.tb01395.x

35. Cronin M, Stanton RM, Francis KP, Tangney M. Bacterial vectors for imaging and cancer gene therapy: a review. Cancer Gene Ther. 2012;19:731-740. doi:10.1038/cgt.2012.59

36. Fu GF, Li X, Hou YY, Fan YR, Liu WH, Xu GX. Bifidobacterium longum as an oral delivery system of endostatin for gene therapy on solid liver cancer. Cancer Gene Ther. 2005;12:133-140. doi:10.1038/ sj.cgt. 7700758

37. Sakaguchi K, He J, Tani S, Kano Y, Suzuki T. A targeted gene knockout method using a newly constructed temperature-sensitive plasmid mediated homologous recombination in Bifidobacterium longum. Appl Microbiol Biotechnol. 2012;95:499-509. doi:10.1007/ s00253-012-4090-4
38. Luo Y, Xu D, Gao X, et al. Nanoparticles conjugated with bacteria targeting tumors for precision imaging and therapy. Biochem Biophys Res Commun. 2019;514:1147-1153. doi:10.1016/j.bbrc.2019.05.074

39. Gao X, Zou W, Jiang B, et al. Experimental study of retention on the combination of bifidobacterium with High-Intensity Focused Ultrasound (HIFU) synergistic substance in tumor tissues. Sci Rep. 2019;9:6423. doi:10.1038/s41598-019-42832-4

40. Jian J, Liu C, Gong Y, et al. India ink incorporated multifunctional phase-transition nanodroplets for photoacoustic/ultrasound dual-modality imaging and photoacoustic effect based tumor therapy. Theranostics. 2014;4:1026-1038. doi:10.7150/thno.9754

41. Hobbs SK, Monsky WL, Yuan F, et al. Regulation of transport pathways in tumor vessels: role of tumor type and microenvironment. Proc Natl Acad Sci USA. 1998;95:4607-4612. doi:10.1073/ pnas.95.8.4607

42. Brigger I, Dubernet C, Couvreur P. Nanoparticles in cancer therapy and diagnosis. Adv Drug Deliv Rev. 2002;54:631-651. doi:10.1016/ S0169-409X(02)00044-3

43. Jin M, Jin G, Kang L, Chen L, Gao Z, Huang W. Smart polymeric nanoparticles with $\mathrm{pH}$-responsive and PEG-detachable properties for co-delivering paclitaxel and survivin siRNA to enhance antitumor outcomes. Int J Nanomedicine. 2018;13:2405-2426. doi:10.2147/IJN

44. Ramazani F, Chen W, van Nostrum CF, et al. Strategies for encapsulation of small hydrophilic and amphiphilic drugs in PLGA microspheres: state-of-the-art and challenges. Int $J$ Pharm. 2016;499:358-367. doi:10.1016/j.ijpharm.2016.01.020

45. Iqbal M, Zafar N, Fessi H, Elaissari A. Double emulsion solvent evaporation techniques used for drug encapsulation. Int J Pharm. 2015;496:173-190. doi:10.1016/j.ijpharm.2015.10.057

46. Ntziachristos V, Tung CH, Bremer C, Weissleder R. Fluorescence molecular tomography resolves protease activity in vivo. Nat Med. 2002;8:757-760. doi:10.1038/nm729

47. Zhou $\mathrm{H}$, He $\mathrm{Z}$, Wang $\mathrm{C}$, et al. Intravenous administration is an effective and safe route for cancer gene therapy using the bifidobacterium-mediated recombinant HSV-1 thymidine kinase and ganciclovir. Int J Mol Sci. 2016;17(6):891.

48. Valluru KS, Wilson KE, Willmann JK. Photoacoustic imaging in oncology: translational preclinical and early clinical experience. Radiology. 2016;280:332-349. doi:10.1148/radiol.16151414
International Journal of Nanomedicine

\section{Publish your work in this journal}

The International Journal of Nanomedicine is an international, peerreviewed journal focusing on the application of nanotechnology in diagnostics, therapeutics, and drug delivery systems throughout the biomedical field. This journal is indexed on PubMed Central, MedLine, CAS, SciSearch ${ }^{\mathbb{B}}$, Current Contents ${ }^{\mathbb{B}} /$ Clinical Medicine, $^{2}$
Journal Citation Reports/Science Edition, EMBase, Scopus and the Elsevier Bibliographic databases. The manuscript management system is completely online and includes a very quick and fair peer-review system, which is all easy to use. Visit http://www.dovepress.com/ testimonials.php to read real quotes from published authors. 